\title{
DISCI.AIMER
}

This report was prepared as an account of work spansorell by an agency of the Uniteid States Guvernuent. Neither the Iniled States Goveratuent nor any agency thesed, nor any of their umployees, miskes any warranty, express or implied, or assumes any legal liahility or respansi-

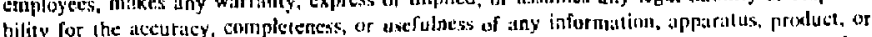

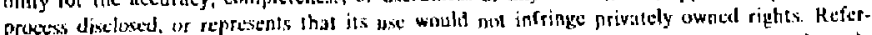

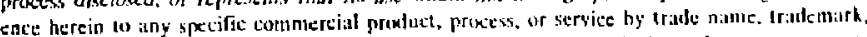

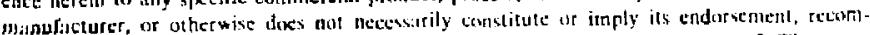

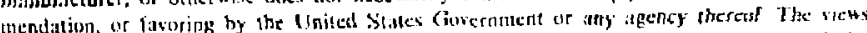

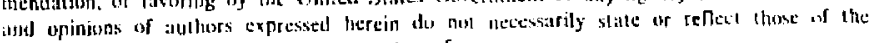
Unted States Gurernment or any agenty thereuf.

\section{A Unified Theory of Tokamak Transport via the Generalized Balescu-Lenard Collision Operator}

\author{
H.E.Myaick and R.E.Duvall \\ Plasma Physics Laboratory, Princeton Liniversity \\ P.O. Box 451 \\ Princeton, New Jersey 08543-0451, U.S.A.
}

\begin{abstract}
A unified basis from which to study the transport of tokamaks at low collisionality is provided by specializing the "generalized Balescu-Lenard" collision operator to toroidal geometry. Explicitly evaluating this operator, ripple, turbulent, and neoclassical transport coefficients are obtained, simply by further specializing the single operator to different particular classes of fluctuation wavelength and mode structure. For each class of fuctuations, the operator possesses a diffusive, test-particle contribution $\mathbf{D}$, and in addition a dynamic drag term $F$, which makes the opesator self-consistent, and whose presence is accordingly essential for the resultant fluxes to possess the appropriate conservation laws and symmetries. These properties, well-known for axisymmetric transport, are demoristrated for one type of turbulent transport, chosen for definiteness, by explicit evaluation of both the "anomalous diffusion" term arising from $\mathbf{D}$, as well as the closely related "anomalous pinch" term coming from $F$. The latter term is neglected by test particle calculations, but is shown to have an important impact on the predicted fluxes.
\end{abstract}




\section{Introduction}

In previous work, 'the action-angle formalism² was used to generalize the Balescu-Lenard (BL) collision operator to its (fully electromagnetic) asaJogue in the space of invariant actions $\mathbf{J} \equiv\left(J_{1}, J_{2}, J_{3}\right)$ of the unperturbed motion. Assuming only that the unperturbed particle motion is integrable, this "generalized-BL" (gBL) operator describes the process of diffusion and

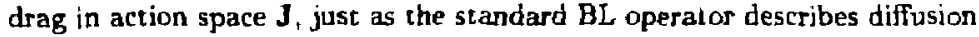
and drag in momentum-space $p$, the invariant actions for the especially simple case of untragnetized motion. The gBL operator is thus easily specialized to a wide range of geometries, including the unmagnetized case, as well as inhomogeneous slabs, cylindrical plasmas, and axisymmetric configurations, simply by assigning a specific physical significance to the actions $J$ and corijugate angles $\theta$. As for the standard $\mathrm{BL}$ operator in the homogeneous, unmagnetized case, the effects of perturbations of all wavelengths appear in the gBL opetator in a uniform way. The gBL operator thus provides a common framework from which to view the effects on transport of perturbations of very short wavelength $\left(\lambda<\lambda_{D}\right)$, which give rise to collisional symmetric ("classical" and "neoclassical") transport, of the longer wavelengths in the turbulent range, and of wavelengths in the macroscopic range of ripple perturhations. In Ref. 1 it was accordingly argued that, along with the quasilinear diffusion tensor ${ }^{2} D(J)$ to describe transport induced by non-self-consistent perturbations, the $\mathrm{GBL}$ operator developed there provides a unifying basis from which to view these three basic mechanisms of tokamak transport, which are normally regarded as different and distinct.

The principal purpose of the present work is to make contact between the formal expressions of Ref, 1 and tokamak physics, by specializing the gBL operator to the case of tokamak geometry, and showing that the formal operator may in fact be explicitly evaluated, how the mechanics of this evaluation proceeds, and what the explicit results look like. In so doing, we more concretely demonstrate two general aspects of the unifying character of the gBL operator. First, it will be seen that, within the action-angle framework, symmetric, turbulent, and ripple transport coefficients emerge as specializations to different particular perturbing spectra of the same general expression. Second, the implications for transport of important properties shown by the $\mathrm{gBL}$ operator to be shared by the different mechanisms can be studied.

One of the most important of these common properties is the effect of 
"self-consistency." i.e., of including the back-reaction of the particles on the fluctuations, in addition to the transport effects of the fluctuations on the particles. As for the standard BL operator, for the gBL operator inclusion of self-consistency means retaining the 'dynamic friction' term $\mathbf{F}$, in addition to the diffusive term $\mathbf{D}$ in the operator. The operator may be written

$$
\begin{aligned}
C f & =-\partial_{\mathbf{J}} \cdot \mathbf{\Gamma} \\
& \equiv \partial_{\mathbf{J}} \cdot\left(\mathbf{D} \cdot \partial_{\mathbf{J}} f-\mathbf{F} f\right) .
\end{aligned}
$$

In general, we shall use the convention that $\partial_{x}$ denotes the partial derivative with respect to any variable $x$, and if $x$ is a vector $(\mathrm{e} . \mathrm{g}, \boldsymbol{x} \rightarrow \mathbf{J}$ ), a gradient in the space of that vector is denoted. $\Gamma$ is a flux in action space, due to the perturbing fields.j As shown in Ref. 1, F is essential for the operator to possess the correct conservation laws, and, in particular, the conservation of toroidal angular momentum $p_{\zeta}$. As observed in Ref. $1_{1}$ and as will be demonstrated here in more concrete form, the constancy of $p_{\zeta}$ implies intrinsic ambipolarity of the transport induced by any portion of the fluctuation spectrum, and thus this property holds for the turbulent as well as the collisional spectrum. For a two species plasma, the turbulent transport is accordingly characterized by properties well-known for symmetric transport ${ }^{3}$ :

1. Interactions between particles of the same species do not produce any net particle transport.

2. The particle fluxes of the two species are equal.

3. The transport is independent of the radial electrostatic potential.

For symmetric transport, it is well-known that violating self-consistenc: by dropping $F$ in $C$ leads to a prediction for the jon particle flux much larger than the electron flux, in contradiction to property (2) here. Similarly, it will be seen that doing the analogous test-particle calculation for turbulent transport results in a prediction for the particle flux of one species which is much larger than the self-consistent result. (Which species depends upon the particular transport mechanism.) As noted from the abstract form in Ref. 1, the turbulent contribution to $F$ represents an "anomalous pinch" term, closely related in form to the "anomalous diffusion" term coming from $D$, which cancels the like-particle portion of the test-particle flux. In this paper, an explicit expression for this anomalous pinch term will be jiven, for one particular turbulent transport mechanism. 
The manner in which self-consistency of the turbulent transport is included in the present work requires some clarification. Entering into the expressions for $\mathbf{D}$ and $\mathbf{F}$ in $\mathrm{Eq}$. (1) is the spectrum of the perturbations inducing the transport. As for the standard BL operator, the spectrum which appears in both $\mathbf{D}$ and $\mathbf{F}$ in the gBL operator is a thermal fluctuation spectrum, which will not properly predict the spectrum of a fully turbulent tokamak. However, the important properties of the $\mathrm{gBL}$ operator follow not from the specific form of the spectrum, but from the related way in which the spectrum appears in $\mathbf{D}$ and F. Thus, in this work, we adopt a "pseudothermal" model for the turbulent fluctuation spectrum, taking literally the thermal structire of the spectrum [cf.Eqs. (90),(91)], but replacing the form of the thermal spectrum with a model spectrum which better represents the spectra of realistic experiments. In so doing one obtains an operator containing a realistic spectrum, while at the same time maintaining the desired conservation laws, $\mathrm{H}$-theorem, etc.

Performing this replacement, but retaining only the term in $\mathbf{D}$ in Eq. (1) is equivalent to doing the quasilinear, test-particle approach followed by a good deal of existing work in turbulent transport. This approach has the virtues of being analycically manageable, and of using fluctuation spectra one regards as properly modelling experjment. However, as already noted, all such approaches are non- self-consistent, with the loss of important properties this implies. On the other hand, more complete theories of turbulence retain self-consistency, but result in formidable complexity of the equations to be solved. (Numerous examples of both approaches may be found in the review article by Liewer. ${ }^{4}$ ) The present treatment. may be regarded as an intermediate approach, retaining the advantages of mathematical simplicity and ease of physical interpretation of the former, while also acquiring important self-consistency properties of the latter, without actually having to compute the turbulent spectrum.

The structure of the remainder of the paper is as follows. In Sec. II, we summarize the formal results which will be needed for the applications to follow. Section III introduces the notation and additional physics necessary to specialize the general expressions of Sec. II to toroidal geometry. In particular, central to the formalism are the "coupling coefficients" $h(1, J, w)$, which succinctly describe the characteristics of the interaction between par: ticles and the perturbations of the system. Abstractly defined in Eq.(6), $h(\mathbf{l}, \mathbf{J}, \omega)$ specialized to toroidal geometry is given in Eq. (33). This single expression contains all three of the basic tokamak transport mecharisms, 
for both electrostatic and magnetic perturbations.

Studying a particulas transport mechanism in this formalism amounts to specializing $h(1, J, w)$ to the relevant class of perturbations inducing the transport, inserting it into the general expressions for $\mathbf{D}$ or $\mathbf{F}$, and performing the necessary summations. In Sec. IV we illustrate this, and with it, the first of the unifying aspects of the formalism, by evaluating $\mathbf{D}$ for three classes of perturbations, one representing each of the three general types of tokamak transport.

Evaluating D alone brings out a good portion of the mechanics involved in the evaluation of the full flux $\boldsymbol{\Gamma}$, but in a somewhat simpler context. In order to further aid clarity, we evaluate $D$ in this section in the "Lorentz limit," where the mass $M_{1}$ of diffusing particles is negligible in comparison with the mass $M_{2}$ of the scattering species. This causes substantial simplifications in the evaluation process. In Sec. $V$, both of these simplifications are removed, evaluating $\Gamma$ for the turbulent mechanism of Sec. IV-B for the cases $M_{3} \ll M_{2}, M_{1} \gg M_{2}$, as well as the case $M_{1} \sim M_{2}$. This permits us to derive explicit expressions for the radial fluxes [cf. Eqs. (98),(101), and (116)] due to all combinations (1-1,1-2,2-1, and 2-2) of species-species interactions, and in so doing, to demonstrate explicitly properties (1-3) of self-consistent transport already cited, for the chosen turbulent transport mechanism. The two terms in the factor $A_{1} / e_{1}-A_{2} / e_{2}$ in each of these expressions for the flux correspond to the diffusive and frictional contributions to $\boldsymbol{\Gamma}$. Thus, the second of these terms is an explicit expression for the "anomalous pinch," whose existence was pointed out in Ref.1, from consideration of the abstract form of the $\mathrm{gBL}$ operator. We conclude Sec. $\mathrm{V}$ by demonstrating that the results obtained obey the Onsager symmetries.

In Sec. VI, we provide some further physical interpretation of the transport results of Sec. V, considering the relative size and scaling of the transport contributions from different species-species interactions. This discussion completes the analogy which exists between symmetric transport and this representative turbulent mechanism. We conclude the section with some summarizing comments.

\section{The Formalism}

Here, we briefly review the abstract results from the action-angle formalism which bear on the transport problem, without reference to any particular magnetic configuration. The essence of the action-angle formalism is the 
reparametrization of the phase point $z$ of a particle from the more directly physical set $(\boldsymbol{r}, \boldsymbol{p})$ of the real-space position $\boldsymbol{r}$ and its conjugate momentum $\mathbf{p}$, to the mathematically more convenient set $(\boldsymbol{\theta}, \mathbf{J})$. Because the $\mathbf{J}$ are constants of the unpercurbed motion, the unperturbed Hamijtonian $H_{0}$ is independent of $\boldsymbol{\theta}$ :

$$
H(z, t)=H_{0}(\mathbf{J})+h(\theta, \mathbf{J}, t)
$$

where

$$
h(z, t)=-\frac{e}{c} \mathbf{v}(z) \cdot \mathbf{A}_{1}[\mathbf{r}(z), t]+e \phi_{1}[\mathbf{r}(z), t]=-\frac{e}{c} v^{\mu} A_{1 \mu}
$$

is the perturbing Hamiltonian. In it, $\boldsymbol{A}_{1}$ and $\phi_{1}$ are the perturbing parts of the vector and electrostatic potentials, respectively. In the second form here, $h$ is written in covariant 4-vector notation for compactness. From Hamilton's equations, one has

$$
\dot{\boldsymbol{\theta}}=\partial_{\mathbf{J}} H=\boldsymbol{\Omega}(\mathbf{J})+\partial_{\mathbf{J}} h
$$

and

$$
\dot{\mathbf{J}}=-\partial_{\theta} h=-i \sum_{\mathbf{I}} \mathbf{l} h(\mathbf{1}, \mathbf{J}, \imath) \exp (i \mathbf{l} \cdot \theta) .
$$

The time-transform $h(1, \mathbf{J}, \omega)$ of the Fourier coefficients of $h(\mathbf{l}, \mathbf{J}, t)$ here are the "coupling coefficients,"

$$
h(\mathbf{l}, \mathbf{J}, \omega) \equiv \oint \frac{d \theta}{(2 \pi)^{3}} e^{-i \mathbf{l} \cdot \theta_{h(z, \omega)},}
$$

which play a central role in the formalism. In Eq. (4), $\Omega \equiv \partial_{\mathbf{J}} H_{0}$ is the unpert urbed time rate of change of $\theta$. Thus, in the absence of $h$, the $\theta$ evolve linearly in time, at rate $\mathbf{\Omega}$. In Eq. (5) $I \equiv\left(l_{1}, l_{2}, l_{3}\right)$ is a thre-component vectcr index, specifying the Fourier harmonic.

When no ambiguity results, we shall abbreviate species label $s_{1}, s_{2}$ with simply the subscripts 1,2 . Unless otherwise indicated, we adopt the convention that a subscript " 1 " refers to the particle or phase point being scattered, and subscript " 2 " refers to scattering particles. The quasilinear diffusion tensor in action-space may then be written ${ }^{2}$

$$
\mathbf{D}_{Q^{l}}(1)=\sum_{a} \sum_{\mathbf{l}_{1}} \mathbf{l}_{1} \mathbf{l}_{1} \pi \delta\left(\mathbf{l}_{1} \cdot \mathbf{\Omega}_{1}-\omega_{a}\right)\left|h\left(\mathbf{l}_{1}, \mathbf{J}_{1}, \omega_{a} ; a\right)\right|^{2}
$$


Here, $a$ is a mode index, labeling each of the coherent perturbations to the system. The diffusive term $\mathbf{D}$ in the $\mathrm{gBL}$ operator is given by

$$
\mathbf{D}(1)=\sum_{\mathbf{2}} \mathbf{D}(1 \mid 2)
$$

where the diffusion of species 1 induced by species 2 is given by

$$
\mathbf{D}(1 ; 2)=\sum_{a} \sum_{\mathbf{l}_{1}} \mathbf{l}_{1} \mathbf{l}_{1} \sum_{\mathbf{l}_{2}}(2 \pi)^{3} \int d \mathbf{J}_{2} f(\mathbf{2}) \pi \delta\left(\mathbf{l}_{1} \cdot \boldsymbol{\Omega}_{3}-\omega_{1}\right)|4 \pi \alpha|^{2} !_{\omega_{1}=\mathbf{l}_{2}-\boldsymbol{\Omega}_{3}} \text {. }
$$

Here, $f(2) \equiv f\left(\mathrm{~J}_{2}\right)$ is the $\theta_{2}$-average of the scattering diatribution, and the coefficjents $\alpha$, defined by

$$
\alpha\left(\mathbf{l}_{1}, J_{1}, \mathbf{l}_{2}, J_{2}, \omega, a\right) \equiv \frac{h\left(\mathbf{l}_{1}, J_{1}, \omega \mid a\right) h^{*}\left(l_{2}, J_{2}, \omega \mid a\right)}{N_{a} \Delta_{a}(\omega)},
$$

measure the effectiveness of mode a in coupling particles 1 and 2 . In $\alpha$, $N_{a} \equiv \int d \mathbf{x}\left|\mathbf{E}_{a}(\mathbf{x})\right|^{2}$ is a normalizing factor, $\mathbf{E}_{a}(\mathbf{x})$ is the electric field for normal mode $a$, and $s_{a}(\omega)$ is the eigenvalue for mode $a$ of the Maxwell operator, ${ }^{1}$ the generalization to inhomogeneous, electromagnetically interacting plasmas of the dielectric function $\epsilon(k, \omega)$ whose square appears in the denominator of the standard BL operator. In Eq. (8), D(1|2) is written in a form where its structural resemblance to $D_{q l}$ is most apparent; the spectrum $|\boldsymbol{h}|^{2}$ in $\mathbf{D}_{q l}$ is replaced in $\mathbf{D}(1 \mid 2)$ by the sum $\sum_{l_{1}}(2 \pi)^{3} \int d J_{2} f(2)|4 \pi \alpha|^{2}$ over contributions to the spectrum from all particles in the scattering distribution. It may also be written in a form more transparently resembling its analogue in the standard BL operator,

$$
\mathbf{D}(1 \mid 2)=(2 \pi)^{3} \int d \mathbf{J}_{2} \mathbf{Q}_{D}(1,2) f(2),
$$

where the diffusive kernel $\boldsymbol{Q}_{D}$ is given by

$$
Q_{D}(1,2) \equiv \sum_{l_{1}, l_{2}} l_{1} l_{1} Q(1,2)
$$

with scalar kernel

$$
\left.Q(1,2) \equiv \sum_{a} \pi \delta\left(\mathbf{I}_{1} \cdot \mathbf{\Omega}_{1}-\mathbf{I}_{2} \cdot \mathbf{\Omega}_{2}\right)|4 \pi a|^{2}\right|_{\omega=I_{2} \cdot \Omega_{2}}=Q(2,1)
$$

Sirrilarly the dynamic friction term $F$ is given by

$$
F(1)=\sum_{2} F(1 ; 2)
$$


where

$$
\mathbf{F}(1: 2)=(2 \pi)^{3} \int d \mathbf{J}_{2} \mathbf{Q}_{F}(1,2) \cdot \partial_{\mathbf{J}_{2}} f(2),
$$

and with the frictional kernel $\mathbf{Q}_{\boldsymbol{F}}$ defined by

$$
Q_{F}(1,2) \equiv \sum_{l_{1}, l_{2}} I_{1} I_{2} Q(1,2)
$$

differing from $Q_{D}$ orly in the replacement $l_{1} l_{1} \rightarrow l_{1} l_{2}$. Using expressions (10) and (13) in the expression in Eq. (1) for the J-space flux $\Gamma$, one finds

$$
\Gamma(1)=\sum_{2} \Gamma(1 \mid 2)
$$

with

$$
-\Gamma(1 ; 2)=\sum_{\mathbf{l}_{1}, \mathbf{l}_{2}} \int d^{6_{2}} Q(1,2) \mathbf{l}_{\mathbf{l}}\left(\mathbf{l}_{1} \cdot \partial_{\mathbf{J}_{1}}-\mathbf{l}_{2} \cdot \partial_{\mathbf{J}_{2}}\right) f(1) f(2) .
$$

Here, $\int d^{6} z_{2} \equiv(2 \pi)^{3} \int d J_{2}$ denotes an integration over the full phase space of species 2 , and so may also be written as $\int d r_{2} \int d \mathbf{p}_{2}$, when this parametrization is convenient.

Closely related to Eq. (15) for $\mathbf{\Gamma}$ is the expression derived in Ref. 1 for the time-rate of change of the total entropy:

$$
\begin{aligned}
\dot{S} \equiv \sum_{1} \dot{S}(1) \equiv & -\sum_{1} \frac{d}{d \ell} \int d^{6} z_{1} f(1) \ln f(1) \\
=\quad & \frac{1}{2} \sum_{1,2} \sum_{l_{1}, l_{2}} \int d^{6} z_{1} \int d^{6} z_{2} Q(1,2) f(1) f(2) \\
& \times\left\{\left(l_{1} \cdot \partial_{J_{1}}-I_{2} \cdot \partial_{\mathbf{J}_{2}}\right) \ln [f(1) f(2)\}\right\}^{2}
\end{aligned}
$$

which is manifestly positive definite, giving zero only when $f(1)$ and $f(2)$ are Maxwellians, $f \sim \exp \left(-H_{0} / T\right)$. Equation (16) will be useful later in Sec. $V$ in considering the (Onsager) symmetries of the transport.

\section{Specialization to Toroidal Geometry}

We now specialize the general formalism of Sec. II to the axisymmetric geometry of a tokamak. We parametrize real-space $r$ by the flux coordinates $(r, \theta, \zeta)$, with minor-radial variable $r$ constant on a flux surface. It is useful 
to use the contravariant basis vectors $e^{i}$, given by $e^{r} \equiv \nabla r \simeq \hat{r}, e^{\theta} \equiv \nabla \theta \simeq$ $\dot{\theta} / r, \mathrm{e}^{\zeta} \equiv \nabla \zeta \simeq \dot{\zeta} / R$, dual to the covariant set $e_{r} \simeq \dot{r}, e_{\theta} \simeq r \dot{\theta}, e_{\zeta} \simeq R \dot{\zeta}$. Thus, one may write wavevector $k$ as

$$
\mathbf{k} \approx k_{i} \mathrm{e}^{i}=k_{r} \Gamma r+m \nabla \theta+n \nabla \zeta,
$$

and the unperturbed vector potential $\mathbf{A}_{0}$ as

$$
A_{0}=A_{0 i} e^{i}=\psi(r) \nabla \theta-\chi(r) \nabla \zeta
$$

i.e., $A_{0}$ has covariant components $\left(A_{0 r}, A_{0 \phi}, A_{0 \xi}\right)=(0, \psi(r),-\chi(r))$. The unperturbed magnetic field is thus given by

$$
\mathbf{B}=\mathbf{B}_{\ell}+\mathbf{B}_{p}=\nabla \psi \times \nabla \theta+\nabla \zeta \times \nabla \mathbf{X},
$$

and the safety factor is $q(r) \equiv d \psi / d \chi$. It will also be useful to employ the right-hand triad of unit vectors $(\dot{\boldsymbol{r}}, \dot{q}, \hat{b})$, where $\dot{b} \equiv \mathbf{B} / \boldsymbol{B}$, and $\dot{q} \equiv \dot{b} \times \dot{\boldsymbol{r}}$, in terms of which one may write $\mathbf{k}=\mathbf{k}_{\|}+\mathbf{k}_{\perp}, \mathbf{k}_{\|} \equiv \hat{b} k_{\|\|}$, and $\mathbf{k}_{\perp} \equiv \dot{\boldsymbol{r}} \boldsymbol{k}_{r}+\dot{q} \boldsymbol{k}_{q}$.

For toroidal geometry, an appropriate specialization of the actions $J$ is $^{1,2.5,6}\left(J_{1}, J_{2}, J_{3}\right) \rightarrow\left(J_{g}, J_{b}, J_{\zeta} \equiv p_{\zeta}\right)$, with $J_{g}$ the gyroaction (equal to $M c / e$ times the usual magnetic moment $\mu$ ), $J_{b}$ the bounce-action (equal to the torojdal flux $\psi$ enclosed by a drift orbit), and $p_{6}$ the toroidal angular momentum,

$$
p_{\zeta} \equiv \mathbf{e}_{\zeta} \cdot \mathbf{p}=\frac{\mathbf{e}}{\mathbf{c}} A_{0 \zeta}+M v_{\zeta} .
$$

The conjugate angles $\theta$ are $\left(\theta_{g}, \theta_{b}, \zeta_{0}\right)$, the gyrophase, the bounce phase, and the bounce-averaged torojdal aximuth, respectively. The set of subscripts $(g, b, \zeta)$ used here in designating the actions $J$ will also be used for related triplet quantities, e.g., $l=\left(l_{g}, l_{b}, l_{\zeta}\right)$.

In $\mathbf{J}$-space, we will have use for the (contravariant) basis vectors $\boldsymbol{\epsilon}^{i} \equiv$ $\partial_{\mathbf{J}} J_{i_{1}}(i=g, b, \zeta)$, which are unit vectors. For any function $F(J)$, we also define $\boldsymbol{\epsilon}^{\boldsymbol{F}} \equiv \partial_{\mathbf{J}} F$, useful in extracting more directly physical information from action-space quantities.

One useful physical function of $\mathbf{J}$ is the particle "banana center" $r_{b}$, the average value of $r$ which a particle has over a bounce period. By considering transport in $r_{b}$ rather than in $r$, one eliminates from the problem the complexities of the unperturbed motion, such as finite gyroradius $\rho_{g}$ and banana width $r_{1}$, which one knows are irrelevant to the net radial step taken per 
bounce in the diffusive motion of the particle, no matter how large they are. Bounce-averaging Eq. (17) and using the constancy of $p_{\zeta}$, one has

$$
p_{\zeta}=\frac{e}{c} \bar{A}_{O \zeta}-M \bar{v}_{\zeta}
$$

For $\tau=0$, one has $\bar{v}_{\zeta}=b_{t} R \bar{v}_{i j}=0$ (where $b_{t, p} \equiv B_{t, p} / B$ ), so one may define $r_{b}$ for this case by

$$
p_{\zeta} \equiv \frac{e}{c} \bar{A}_{0 \zeta}\left(r_{b}\right) \text {. }
$$

For $\tau=1, p_{\zeta}$ in Eq. (18) acquires a kinetıc portion, while $J_{b}$ becomes a purely minor radial variable. ${ }^{2,5,6}$ The appropriate definition of $r_{b}$ in this case is

$$
J_{b} \equiv \frac{e}{c} \bar{A}_{\infty 0}\left(r_{b}\right) \text {. }
$$

Thus, one has

$$
\epsilon^{r} \equiv \partial_{J} r_{b}= \begin{cases}-\epsilon^{\zeta} / M f \Omega_{g} R b_{p} & (\tau=0) \\ \epsilon^{b} / M \Omega_{g} r b_{t} & (\tau=1) .\end{cases}
$$

For $\tau=0, J_{b}$ is $v_{i \mid}$-like,

$$
J_{b}=(2 \pi)^{-1} \oint d s_{i \mid} M v_{\|}=(2 \pi)^{-1} \oint d \theta g M v_{\zeta} \equiv q M \bar{v}_{\zeta},
$$

while for $\tau=1$, one readily shows from Eqs. (20) and (18) that ${ }^{8}$

$$
J_{v} \equiv J_{b}-\frac{e}{c} \psi\left(x=-\frac{c}{e} p_{\zeta}\right)=q M \bar{v}_{\zeta} .
$$

Thus, extending this $\tau=1$-definition for $J_{v}$ to $\tau=0$ by $J_{v}(\tau=0) \equiv J_{b}$, we can extract bounce-averaged $v_{\|}$information from the $\mathbf{J}$ using

$$
\epsilon^{J_{v}} \equiv \partial_{\mathbf{J}} J_{v}= \begin{cases}\epsilon^{b} & (\tau=0), \\ \epsilon^{b}+q \epsilon^{6} & (\tau=1) .\end{cases}
$$

\section{A. The Coupling Coefficients}

Fundamental to the calculation of $\mathbf{D}$ are the coupling coefficients $h(l, J, \omega \mid a)$. These have been worked out for various cases previously. ${ }^{5,7}$ Here, we give a more complete evaluation. The $h(I, J, w \mid a)$ require a description of the spatial structure of each contributing mode $a$, and of the unperturbed particle motion. Fully describing the strncture of the modes 
in a tokamak is a field in itself. We shall content ourselves with a model description, chosen to satisfy a number of the general important chas acteristics which one knows the modes should possess. Our model for the mode structure for all components $A_{\alpha \mu}(\mu=1, \ldots, 4)$ of mode $a$ is the eikonal form

$$
\left.A_{a_{\alpha}}(x)=\bar{A}_{a_{\mu}}(r) \exp i: \int d r^{\prime} k_{r}\left(r^{\prime}\right)+m \theta \div \pi \zeta\right) .
$$

The toroidal "quantum number" $n$ is rigorously constant, due to axisymmetry, while taking $m$ constant is only approximate, due to toroidal effects. $k_{r}(r)$ is the radial wave number, and $\bar{A}_{\sigma \mu}(r)$ is the mode amplitude. We shall assume here that $k_{r}$ and $\vec{A}_{\mathrm{a} \mu}(r)$ may be assumed about constant over the minor-radial excursion of the unperturbed orbit of the particle in question. (We emphasize that situations for which this assumption is not valid present no difficulty for the basic formalism; the integrals to be performed are simply somewhat different.) Thus, over a particle's orbit, mode $a$ is characterized by a loral wavevector $\mathbf{k}\left(r \simeq r_{b}\right)$. For externally imposed perturbations, such as ripple from the toroidal-field coils, $\bar{A}_{\alpha \mu}$ typically falls off as one moves radially inward. For internally generated perturbations, a reasonable model is taking $\bar{A}_{a \mu}$ localized about some minor radius $r_{a}$, with localization width $w_{a}$. We choose the simplified form

$$
\bar{A}_{a \mu}=\bar{A}_{\alpha \mu} s\left(w_{a} / 2, \tau-r_{a}\right),
$$

where $s(x, y)$ is a step-like localizing function, defined as

$$
s(z, y) \equiv \begin{cases}1 & (x \geq|y|) \\ 0 & (x<|y|) .\end{cases}
$$

(Here, and in what follows, the double overbar will be used to denote the amplitude of quantities with this radial localization expjicjtly displayed.) in addition to being radially localized, the set of modes $a$ are to represent a full set of eigenmodes of the Maxwell operator, which, owing to the (near) Hermiticity of that operator, one expects to be orthogonal and complete. We incorporate this general property into our model by envisioning each mode $a$ to be specified by both wavevector $k$, and by localization radjus $r_{a}$. Thus, modes with the same $k$ are localized within sequential nested toroidal shells, each of thickness $w_{a}$, centered at radii $r_{a 1}, r_{a 2}, \ldots$, and having volume $V_{a} \simeq\left(2 \pi r_{a i}\right)(2 \pi R) w_{a}$, jointly comprising the plasma volume $V_{p}=\sum r_{a} V_{a}$. Therefore, the sum over $a$ in $Q(1,2)$ becomes a sum $\Sigma_{\tau_{\mathbf{a}}} \Sigma_{\mathbf{k}}$ - 
We describe the particle motion as in Ref.5. We make the usual separation of $r$ isto contrjbutions from the guiding-center motion and the gyromotion,

$$
\mathbf{r}(z)=\mathbf{R}+\boldsymbol{\rho}_{\mathrm{g}}
$$

The giromotion is given by

$$
\rho_{g}\left(\theta_{g}\right)=\rho_{g}\left(\dot{r} \cos \theta_{g}-\dot{q} \sin \theta_{g}\right),
$$

with $\rho_{g} \equiv v_{\perp} / \Omega$ the gyroradius. The guiding-center motion is described by

$$
\mathbf{R}\left(\theta_{b}, \zeta_{0}\right)=\left(e_{r} r_{b}+e_{g} \tau \theta_{b}+e_{\zeta} \zeta_{0}\right)+\rho_{b},
$$

where the oscillatory portics of the bounce motion is given by

$$
\rho_{b}\left(\theta_{b}\right) \equiv \mathbf{e}_{r} r_{1} \cos \theta_{b}+\left(\mathbf{e}_{\theta} \theta_{1}+\mathbf{e}_{\zeta} \zeta_{1}\right) \sin \theta_{b} .
$$

Here, $r_{b}$ is the particle banana center, constant in time. The canonical phases $\theta=\left(\theta_{g}, \theta_{b}, \zeta_{0}\right)$ evolve linearly in time, as discussed in Sec. II. The particle velocity, needed in Eq. (3), is thus obtained from Eq. (27) by

$$
\mathbf{v}(z) \equiv \dot{\mathbf{r}}(z)=\Omega_{i} \partial_{\theta,} \mathbf{r}(z)
$$

The time-independent amplitudes $r_{1}, \theta_{1}$, and $\zeta_{1}$ measure the size of the particle excursions in the $r, \theta$, and $\zeta$-directions in the course of a bounce (or transit) period $\tau_{b}$. Trapping-state index $\tau$, already defined, provides the secular contribution to $\theta_{b}$ for passing particles, and none for trapped particles, as is appropriate.

The use of only the fundamental harmonics $\cos \theta_{b}$ and $\sin \theta_{b}$ in expression (30) makes it strictly valid only for particles not too near the trapped/passing boundary. The coupling coefficients for particles near this boundary involve integrals which yield funetions less standard than those from the "harmonic approximation" of Eq. (30). However, the qualitative behavior is not much changed.

Using expressions (24)-(30) and Eq. (6), the evaluation of $h(1, J, w \mid a)$ is straightforward, using the Bessel identity

$$
J_{l}(z)=\oint \frac{d \theta}{2 \pi} e^{-i l \theta} e^{i z \sin \theta} .
$$


One finds

$$
\begin{aligned}
& h(\mathbf{l}, \mathbf{J}, \omega)=\delta\left(l_{\zeta}-n\right) e^{-i\left(l_{\mathrm{b}}-\tau m\right) \theta_{4 k}-i l_{g} \theta_{\mathrm{g} k}}\left[e \phi_{J_{1}}, J_{l_{0}-m}\right. \\
& -\left(\frac{e}{c} v_{-} A_{-}\right) \frac{1}{2}\left(J_{l_{g}-1} e^{i \theta_{0 k}-i \theta_{1} A}+J_{l_{-}-1} e^{-i \theta_{g k}+i \theta_{0} A}\right) J_{l_{b}-\pi m} \\
& -\left(\frac{e}{c} u_{1} A\right) J_{l}, J_{l_{b}-\tau m} \\
& -\left(\frac{e}{c} u_{0} A\right) J_{l} \frac{1}{2}\left(J_{l_{k}-\tau m-1} e^{i \dot{b}_{b k}-i \theta_{b A}}+J_{-\tau m+1} e^{-i \theta_{b k}+i \theta_{b A}}\right) .
\end{aligned}
$$

First, we define some terms in this expression, and then provide some interpretation. In it, mode label $a$ has been suppressed. All of the perturbing potentials $\phi$ or $A$ appearing in (33) denote the amplitudes $A\left(r_{B}, m, n\right)$, obtained by setting $r=r_{b}, \theta=0$ and $\zeta=0$ in $A(x)$ in Eq. 24. Each Bessel function $J_{l}(z)$ with $l_{g}$ as its index $l$ has argument $z=z_{g} \equiv k_{\perp} \rho_{g}$, while each $J_{l}$ having bounce-harmonic $l_{b}$ in its index has argurnent $z=z_{b} \equiv$ $\left[\left(k_{\tau} r_{1}\right)^{2}+\left(m \theta_{1}+n \zeta_{1}\right)^{2}\right]^{\frac{1}{2}}$. ( $z_{b}$ is called $y_{1}$ in Ref.5) $A_{\perp}$ in (33) is that component of $\mathbf{A}$ normal to $b$, while $A=|A|$, and $A_{\theta, 6} \equiv \mathbf{e}_{\theta, 6} \cdot \mathbf{A} . \theta_{g A}$ is the gyrophase at which $\mathbf{v}_{\perp}$ is parallel to $\mathbf{A}_{\perp}$, i.c., $\mathbf{v}_{\perp} \cdot \mathbf{A}_{\perp}=v_{\perp} A_{\perp} \cos \left(\theta_{g}-\theta_{g A}\right)$, and $\theta_{g k}$ is defined by $k \cdot \rho_{g}=z_{g} \sin \left(\theta_{g}-\theta_{g k}\right)$, the gyrophase where $v_{\perp}$ is parallel to $\mathbf{k}_{\perp}$. Analogously, we define the phase $\theta_{b A}$ and velocity $u_{0}$ by $\dot{p}_{b} \cdot \mathbf{A}=u_{0} A \cos \left(\theta_{b}-\theta_{b A}\right)$, and $\theta_{b k}$ by $\mathbf{k} \cdot \boldsymbol{p}_{b}=z_{b} \sin \left(\theta_{b}-\theta_{b k}\right)$. The velocity $u_{1}$ is defined as $u_{l} A=\tau \Omega_{b} A_{\theta}+\Omega_{\zeta} A_{\zeta}$. The term in $u_{0,(1)}$ dominates for particles with $\tau=0(1)$.

One first notes the overall factor $\delta\left(l_{\zeta}-n\right)$ multiplying the factor in square brackets in Eq. (33), a consequence of axisymmetry, used in Hef. 1 in the demonstration of $p_{6}$-conservation. The factor in square brackets is a sum of four terms, each coming from a different portion of the inner product $v^{\mu} A_{\mu}$ giving $h$; the first is the electrostatic contribution, from the $\mu=4-$ component, the second is the contribution $v_{\perp} \cdot A_{\perp}$ due to the gyromotion, the third is from that part of the guiding-center contribution $\dot{\mathbf{R}} \cdot \mathbf{A}$ evolving secularly in time, and the fourth is from the part of $\mathbf{R} \cdot \mathbf{A}$ which is oscillatory in time. The structure of each term is the same; each has an amplitude with units of $(e / c) v A$ (hence energy), times a factor involving Bessel functions $J_{l}\left(z_{g}\right)$ coming from the $\theta_{g}$-integration, times a factor involving $J_{l}\left(z_{b}\right)$ arising from the $\theta_{b}$-integration. The gyro- and bounce- related Bessel functions are the strength of that portion of mode a which is oscillatory at exp $i\left(l_{g} \theta_{g}+l_{b} \theta_{b}\right)$, just as the overall factor $\delta\left(l_{\zeta}-n\right)$ gives that portion (namely, all or none) of the mode which is oscillatory at $\exp i l_{\zeta_{0}} \zeta_{0}$. 
In contrast to the all-or-none dependence on $l_{\zeta}$ of the contribution of a given mode, one notes that a mode contributes over a range of $l_{g}$ and $l_{b}$. One recalls the asymptotic forms for the $J_{l}$,

$$
\begin{aligned}
& J_{l}(z) \simeq(z / 2)^{|l|} / \mid l ! ! \\
& J_{l}(z) \simeq(2 / \pi z)^{\frac{1}{2}} \cos (z-l \pi / 2-\pi / 4),(|l|<z) .
\end{aligned}
$$

We consider $J_{l}$ as a function of $l$. One sees that for $l !<z_{l}, J_{l}$ has an l-independent amplitude $(2 / \pi z)^{\frac{1}{2}}$, times a factor oscillatory in $l$. For $|l|>z, J_{l}$ falls off rapidly to zero. Thus, the gyro-related Bessel functions contribute over a range $\Delta l_{g} \sim 2 z_{g}$ about $l_{g}=0$, while the bounce-related ones contribute over a range $\Delta l_{b} \sim 2 z_{b}$ about $l_{b}=\tau \mathrm{m}$.

Because it is helpful in physically interpreting these expressions, and also because it will be useful in making analytic progress in what follows, we pursue this examination of the asymptotic forms of the $J_{l}$ a bit further. Applying the method of stationary phase to approximately evaluate Eq. (32), one obtains Eq. 35 for $\mid l<z$, and $J_{l} \simeq 0$ in place of Eq. (34) for $|l|>z$. The points $\theta_{0}$ of stationary phase, from which the dominant contributions to the integral come, are given by

$$
l=z \cos \theta_{0} .
$$

From this, one may ascribe a particular position $\theta_{90}$ and $\theta_{60}$ on a particle's orbit from which each $h(1, J, w)$ arises. Thus, in contrast to the unmagnetized case, where a particle is resonant or nonresonant with a given mride for all time, for the time-varying velocities in magnetized geometries, a particle passes through a series of local regions at which the variation of a given mode is as exp il - $\mathbf{\Omega} t$, for a surcession of values of $\mathrm{l}$. This is the physical significance of the range of $\mathbf{I}$ contributing to $\mathbf{D}$.

From Eq. (33), one discems the common origin of the three types of tokamak transport. Axisymmetric (collisional) transport is caused by shortwavelength electrostatic perturbations. Thus, it arises from the first term in square brackets in Eq. (33). The electrostatic portion of turbulent transport also comes from this term, while the magnetic portion, arising from modes which tend to have $A_{\|} \gg A_{\perp}$ comes from the third and fourth terms. Finally, as will be seen for longer - wavelength modes, which leave $\mu$ invariant, the second term yields just the $\mu B_{1}$ - ripple perturbation which is the origin of magnetic ripple transport. ( $B_{1}$ is the perturbation of the magnetic field strength $B$.) The first term provides the electrostatic contribution to ripple 
transport. In the following section, we use this singie coupling coefficient to derive expressions for transport coefficients for each of the three basic tokamak transport mechanisms.

\section{Evaluation of D}

In this section, we illustrate the first aspect of the unifying character of the action-angle formalism referred to in the Introduction, evaluating th: diffusion tensor $\mathbf{D}$ for each of symmetric, turbulent, and ripple transport mechanisms, simply by specializing the same expressions for $\mathbf{D}$ and $h(1, J, w)$ to the context relevant to the mechanism of interest.

\section{A. Axisymmetric Collisional Transport (Banana Regime)}

We begin with transport induced by the shortest wavelengths $\left(\lambda<\lambda_{D}\right)$, viz., axisymmetric "banana" transport. It is instructive to consider first the transport of electrons (species 1) scattering off ions (species 2) in the Lorentz limit $M_{1} / M_{2} \rightarrow 0$. From the symmetry of $C f$ under interchange of species label, performing this calculation is essentially the same as treating the opposite limit $M_{1} / M_{2} \rightarrow \infty$ (cf.Sec. V). The evaluation in the more general case $M_{1} \sim M_{2}$ can also be performed analytically, as will be seen in Sec. V. A fuller treatment of the non-Lorentz case will be given elsewhere. ${ }^{9}$ Because the $\delta\left(l_{\zeta}-n\right)$ in the $h(1, J, \omega)$ insures that $l_{1 \zeta}=l_{2 \zeta}=n$, and because the $E \times B$-drift is species independent, the $E \times B$-contribution $\Omega_{C E}$ to the toroidal precession frequency in the argument $\Omega_{\text {ret }} \equiv \mathbf{l}_{\mathbf{3}} \cdot \boldsymbol{\Omega}_{\mathbf{1}}-\mathbf{l}_{\mathbf{2}} \cdot \boldsymbol{\Omega}_{\mathbf{2}}$ of the delta-function in Eq. (12) drops out. Referring to the remaining portion of the particle frequencies $\boldsymbol{\Omega}$ as $\boldsymbol{\Omega}^{\prime}$, in the Lorentz limit, one may take

$$
\mathbf{l}_{\mathbf{2}} \cdot \boldsymbol{\Omega}_{\mathbf{2}}^{\prime} \rightarrow 0
$$

in Eq. (12), for those $l_{2}$ having appreciable $h(1, J, w)$. Keeping only the first (electrostatic) term in Eq. (33), and taking $\Delta_{a}(\omega)$ to its short-wavelength, unshielded limit $\Delta_{c} \rightarrow 1$, one has

$$
N_{\mathrm{a}} \Delta_{\mathrm{a}}=k^{2} V_{\mathrm{a}}
$$

and thus,

$$
\begin{aligned}
& |\alpha|^{2}=\left|e_{1} e_{2} / k^{2} V_{a}\right|^{2} s\left(w_{a} / 2, r_{b 1}-r_{a}\right) s\left(w_{a} / 2, r_{b 2}-r_{a}\right) \times \\
& \delta\left(l_{1 \zeta}-n\right) \delta\left(l_{2 \zeta}-n\right) J_{l_{1}}{ }^{2} J_{l_{16}-r_{1} m^{2}} J_{l_{2 q}}{ }^{2} J_{l_{21}-r_{2 m}}{ }^{2} .
\end{aligned}
$$


Here, $r_{b 1,2} \equiv r_{b}\left(J_{1,2}\right)$. Using this in Eqs. (10-12), one finds

$$
\begin{aligned}
& D(1 \mid 2)=\int d^{6} z_{2} f(2) \sum_{a} \sum_{l_{1}, l_{1}, l_{z_{q}}, l_{2 \downarrow}} l_{1} l_{1} \pi \delta\left(l_{1} \cdot \Omega_{1}^{\prime}\right)\left|\frac{4 \pi e_{1} e_{2}}{k^{2} V_{a}}\right|^{2} \times \\
& s\left(w_{a} / 2, r_{b 1}-r_{a}\right) s\left(w_{a} / 2, r_{b 2}-r_{a}\right) J_{l_{1}}{ }^{2} J_{l_{2 b}-r_{1} m^{2} J_{l_{a}}{ }^{2} J_{l_{2}-r_{2} m}{ }^{2} l_{2 \zeta}=l_{26}=n} \\
& =\sum_{a} \sum_{l_{b a}, l_{1 b}} 1_{1} \mathbf{l}_{1} \pi \delta\left(\mathbf{l}_{\mathrm{l}} \cdot \boldsymbol{\Omega}_{1}^{\prime}\right) s\left(w_{a} / 2, r_{b 1}-r_{a}\right)\left|\frac{4 \pi e_{1} e_{2}}{k^{2} V_{a}^{r}}\right|^{2} \times \\
& {\left[V_{a} n_{2}\left(r_{0}\right)\right] J_{l_{1}}{ }^{2} J_{l_{1 b}-r_{2} m^{2}}} \\
& =V_{a}^{-1} \sum_{\mathbf{k}} \sum_{l_{1,}, l_{1 l}} l_{l} l_{1} \pi \delta\left(\jmath_{l} \cdot \Omega_{l}^{\prime}\right) \mid \frac{4 \pi e_{1} e_{2}}{k^{2}} i^{2} n_{2}\left(r_{b 1}\right) d_{l_{1}},{ }^{2} J_{l_{10}-r_{1} m^{2}} \text {. }
\end{aligned}
$$

In moving from the first to the second form given for $\mathbf{D}$ here, we first note that the only dependence on $\mathbf{I}_{\mathbf{2}}$ in the first form lies in the Bessel functions

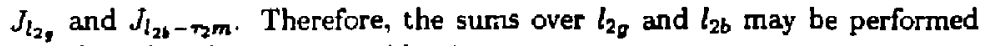
exactly, using the important identity

$$
1=\sum_{l=-\infty}^{\infty} J_{l}^{2}(z) .
$$

The remaining integration $\int d^{6} z_{2}=(2 \pi)^{3} \int d J_{2}=\int d \mathbf{r}_{2} \int d \mathbf{p}_{2}$ over phase space yields the factor $\left[V_{a} \pi_{2}\left(r_{a}\right)\right]$, with $n_{2}\left(r_{a}\right)$ the density of species 2 , averaged over the volume $V_{a}$ around $r_{a}$. In going from the second to the final form, we have performed the sum over $r_{a}$, yielding a nonzero contribution from only that volume $V_{a}$ in which $r_{b l}$ lies.

As usual, the delta-function present in Eq. (38) is to be interpreted not as strictly singular, but as broadened about the resonant surfaces (whert $\boldsymbol{l}_{1} \cdot \boldsymbol{\Omega}_{1}^{\prime}=0$ ), with resonance widths large enough that, given the density of resonances from the sums over $l_{1 g}, l_{16}$ and mode index $a$ (or $\mathbf{k}$ ) in (38), the resonances overlap. Then these sums may be converted to iriegrals.

We perform the integrations, first over the $\left(l_{g}, l_{b}\right)$-plane, and then over k. (Since all the $\mathbf{l}_{2}$-dependence has been eliminated, for brevity we here drop the subscript on $\left.l_{1}\right)$. It is convesient to reparametrize the $\left(l_{g}, l_{b}\right)$-plane with $\omega_{g} \equiv \Omega_{g} l_{g}, \omega_{b} \equiv \Omega_{b} l_{b}$. The integration (summation) to be performed is illustrated in Fig. 1. As seen there, over the $\left(\omega_{g}, w_{b}\right)$-plane, the sum (or integral) in (38) is nonzero along a line with slope -1 , given by the resonance condition

$$
0=\mathbf{l} \cdot \boldsymbol{\Omega}_{1}^{\prime}=\omega_{g}+\omega_{b}+\pi \Omega_{\zeta}^{\prime}
$$


and is appreciable only where the $J_{l}$ are appreciable; hence, in a rectangle centered about $\left(\omega_{g}, \omega_{b}\right)=\left(0, \pi m \Omega_{b}\right)_{1}$ of width $\Delta \omega_{g}$ and height $\Delta \omega_{b}$, defined by

$$
\Delta w_{g} / \pi \equiv z_{g} \Omega_{g} \equiv k_{\perp} v_{-}, \Delta w_{b} / \pi \equiv z_{b} \Omega_{b} \simeq k_{i} \delta v_{!}-k_{\perp} v_{B} .
$$

Here, $v_{B} \simeq\left(\rho_{g} / 2 R\right) v_{\perp}$ is the amplitude of the 'grad-B' drift, normal to $\bar{b}$, and $\delta t_{\|}$is the amplitude of the oscillations in $v_{\|}$over a bounce period. Physically, $\Delta \omega_{g,(b)}$ represents the range of frequencies a particle moving through perturbation a encounters due to its gyro-(bounce-) motion over the course of its orbit. (The reason for the factor of $\pi$ in the definition will become apparent shortly.) For modes having $k_{\perp} \sim k_{\mid \text {, }}$, typical of this section, the last term in Eq. (41), proportional to $v_{B}$, is negligible. However, when $k_{\perp} \gg k_{i \mid}$, as is typical of the turbulent spectrum, to be considered in Sec. B, this tersm is important.

For th: short wavelengths of relevance to this section, one has $z_{b, g} \gg 1$, so many bounce and gyro-harmonics are contained in this rectangle. We therefore approximate both the bounce-and gyro-related factors $J_{l}^{2}$ by the pairwise average of the asymptotic forms for $J_{l}^{2}$ discussed following Eq. (35), which eliminates the oscillatory character from the sums:

$$
\begin{aligned}
\bar{J}_{l}^{2}(z) & \equiv \frac{1}{2}\left[\left(J_{l}^{2}(z)+J_{l+1}^{2}(z)\right]\right. \\
& =s(\pi z / 2, l) /(\pi z) .
\end{aligned}
$$

This gives the correct small- and large-l limits previously discussed, and the coefficient $\pi / 2$ of $z$ in (42), determining the precise transition point from the small- to large-l regimes, is fixed by requiring that (42) satisfy the averaged counterpart of jentity (39).

We now use the approximation (42) in evaluating (38). With $\Sigma_{\mathbf{k}} \rightarrow$ $l_{\alpha}(2 \pi)^{-3} \int d k$, one has, ior any component $D^{i j} \equiv \epsilon^{i} \cdot \mathbf{D} \cdot \epsilon^{j}$ of $\mathbf{D}$,

$$
\begin{aligned}
D^{i j}(1 \mid 2)= & \int \frac{d k}{(2 \pi)^{3}}\left|-\frac{4 \pi e_{1} e_{2}}{k^{2}}\right|^{2} n_{2}\left(r_{b 1}\right) \int_{-\infty}^{\infty} d \omega_{b} \int_{-\infty}^{\infty} d \omega_{g} l_{i} l_{j} \pi \delta\left(\mathbf{1} \cdot \mathbf{\Omega}_{1}^{\prime}\right) \times \\
& \frac{s\left(\Delta \omega_{b} / 2, \omega_{b}^{\prime}\right)}{\Delta \omega_{b}} \frac{s\left(\Delta \omega_{g} / 2, \omega_{a}\right)}{\Delta \omega_{g}} \\
= & \int \frac{d \mathbf{k}}{(2 \pi)^{3}} \pi\left|\frac{4 \pi e_{1} e_{2}}{k^{2}}\right|^{2} n_{2}\left(r_{b 1}\right) \int_{-\infty}^{\infty} d \omega_{b} l_{j} l_{j} \frac{s\left(\Delta \omega_{b} / 2, \omega_{b}^{\prime}\right)}{\Delta \omega_{b}} \frac{s\left(\Delta \omega_{g} / 2, \omega_{g 0}\right)}{\Delta \omega_{g}},
\end{aligned}
$$

where $\omega_{b}^{\prime} \equiv \omega_{b}-\tau m \Omega_{b}$, and $\omega_{g 0}\left(l_{b} n\right) \equiv-\left(\omega_{b}+n \Omega_{\zeta}^{\prime}\right)$. For both $\tau=0$ and $1, \Delta \omega_{b} / \Delta \omega_{g} \lesssim \epsilon^{\frac{1}{2}} k_{l i} / k_{1}$, i.e., the contributing rectangle in the $\left(\omega_{g}, w_{b}\right)-$ 
plane is short and broad, for most $\mathbf{k}$. Thus, we may approximately rantace $l_{b}$ in Eq. (43) by its value $i_{b} \equiv \tau \mathrm{m}$ at the vertical midpoint of the rectangle. This replacement in $\omega_{g 0}$ makes the second (gyro-related) $s$-factor in (43) independent of $\omega_{b}$, rendering the remaining $w_{b}$-integration trivial, $\int_{-\infty}^{\infty} d w_{b} s\left(\Delta w_{b} / 2, w_{b}^{\prime}\right) / \Delta w_{b}=1$. :This corresponds to using the identity (39): This leaves

$$
D^{i j}(12)=\int \frac{d \mathbf{k}}{(2 \pi)^{3}} \pi \bar{l}_{i} \bar{l}_{j}\left|\frac{4 \pi e_{1} e_{2}}{k^{2}}\right|^{2} n_{2}\left(r_{b 1}\right) \frac{s\left[\Delta \omega_{g} / 2, \omega_{g 0}\left(I_{b}, n\right)\right.}{\Delta \omega_{g}},
$$

where

$$
\left.\overline{\mathbf{I}} \equiv\left(\bar{l}_{g}, \bar{i}_{b}, \bar{l}_{\zeta}\right) \equiv\left[-\left(\bar{l}_{b} \Omega_{b}\right)+\bar{i}_{\zeta} \Omega_{\zeta}^{\prime}\right) / \Omega_{g}, \tau m, n\right]
$$

is the averaged value of 1 , having now performed the sum $\Sigma_{l}$. The value of $\bar{l}_{g}$ is a consequence of $(40)$. As a result of this, one notes that there are really only three independent components of $\mathbf{D}$, namely $D^{b 6}, D^{\alpha}$, and $D^{66}$, the rest being given in terms of these by

$$
\begin{aligned}
& D^{g g}=\left(\Omega_{b}^{2} D^{b b}-2 \Omega_{b} \Omega_{\zeta}^{\prime} D^{b \zeta}+\Omega_{\zeta}^{2} D^{\zeta \zeta}\right) / \Omega_{g}^{2}, \\
& D^{g b}=D^{b g}=-\left(\Omega_{b} D^{b b}+\Omega_{\zeta}^{\prime} D^{b \zeta}\right) / \Omega_{g}, \\
& D^{g \zeta}=D^{\zeta g}=-\left(\Omega_{b} D^{b \zeta}+\Omega_{\zeta}^{\prime} D^{\zeta \zeta}\right) / \Omega_{g} .
\end{aligned}
$$

From Eqs. (46) it follows that

$$
\boldsymbol{\Omega}_{1}^{\prime} \cdot \mathbf{D}=\mathbf{D} \cdot \boldsymbol{\Omega}_{1}^{\prime}=0 .
$$

Thus, since $\epsilon^{E^{\prime}} \equiv \partial_{\mathrm{J}} H_{0}^{\prime} \equiv \Omega^{\prime}$ (where $H_{0}^{\prime} \equiv H_{0}-P_{\zeta} \Omega_{\zeta E}$ is the particle energy in the frame precessing at $\Omega_{\zeta E}$ ), the diffusive portion alone of $C$ conserves energy in this precessing frame, in the Lorente approximation. (We shall see that the contribution $F$ to $C$ also conserves $E^{\prime}$ separately in the Lorentz approximation. For the non-Lorentz case, only the contributions from $D$ and $\mathbf{F}$ together, summed over species, conserve energy.$^{9}$ )

One notes from relations (46) the symmetric way in which diffusion in velocity space and real space enter the theory in the actjon-angle framework. This is in some contrast with more standard treatments, ${ }^{3,10}$ where radial diffusion appears as a secondary consequence of diffusion in $\mathbf{v}$.

We now complete the evaluation of $D^{b 6}, D^{b 6}$, and $D^{66}$ by performing the integration over k. Using $n \equiv R k_{t}=R\left(k_{\|} b_{t}-k_{q} b_{p}\right), m \equiv r k_{p}=$ $r\left(k_{\|} b_{p}+k_{q} b_{t}\right)$, the remaining integrals are easily evaluated. For $\tau=0, \omega_{g 0}=$ $-n \Omega_{\zeta} \ll k_{\perp} v_{\perp}$, so that the gyro-related s-function $s\left(\pi k_{\perp} v_{\perp} / 2, \omega_{g 0}\right) \simeq$ 
$s\left(\pi k_{\perp} v_{\perp} / 2,0\right)=1$, for essentially all $\mathbf{k}$. For $T=1, w_{g 0}=-\left(m \Omega_{b}+n \Omega_{\zeta}\right)=$ $-k_{\|} \bar{v}_{\|\|}, \quad\left(\bar{v}_{\|}\right.$is the bounce-averaged parallel velocity $)$, so that $s\left(\pi k_{\perp} v_{\perp} / 2,-k_{\|} \bar{v}_{\| \mid}\right)=s\left(\pi v_{\perp} / 2 \bar{v}_{\|}, k_{\|} / k_{\perp}\right)$ in Eq.(44) selects contributions only from those $k$ having $\alpha_{k} \mid<a_{u}$, where

$$
\tan \alpha_{k} \equiv k_{\|} / k_{\perp}, \tan \alpha_{v} \equiv \pi v_{\perp} / 2 \tilde{u}_{\|} .
$$

As a passing particle approaches the trapped/passing boundary, $\bar{v}_{\|}$goes to 0 , and the domain prescribed by the $s$-function for $\tau=1$ goes smoothly to that for $\tau=0$, making the $D^{i j}$ continuous across the $r=0 \rightarrow 1$ transition.

Physically, for the local resonance discussed around $\mathrm{Eq}_{\mathrm{q}}(36)$, one needs $0=\mathbf{k} \cdot \mathbf{v}=k_{\|} v_{\|}+k_{\perp} v_{\perp} \cos \theta_{g}$, or

$$
\tan a_{k}=-v_{\perp} \cos \theta_{g} / v_{11} .
$$

Because $v_{\|}$goes through 0 for trapped particles, resonance occurs for all values of $\alpha_{k}$ in the interval $[-\pi / 2, \pi / 2]$, for some $\theta_{g}$. For passing particles, however, (49) implies $\left|\tan \alpha_{k}\right|$ is always smaller than some maximum $\left|v_{\perp} / v_{\|}^{\min }\right|$, hence such particles cannot resonate with modes having $\left|k_{\|} / k_{\perp}\right|$ too large.

Using the required $\mathbf{k}$-integrals

$$
\int d \mathbf{k} \frac{s\left(\alpha_{u}, \alpha_{k}\right)}{k_{\perp} k^{4}}\left[\begin{array}{c}
k_{\|}^{2} \\
k_{\perp}^{2} \\
k_{\|} k_{\perp}
\end{array}\right]=\pi^{2} \ln \Lambda\left[\begin{array}{c}
I_{-}\left(\alpha_{v}\right) \\
I_{+}\left(\alpha_{v}\right) \\
0
\end{array}\right]
$$

where $I_{上}\left(\alpha_{v}\right) \equiv \frac{2}{n}\left(\alpha_{4}=\frac{1}{2} \sin 2 \alpha_{v}\right)$, one finds

$$
\left[\begin{array}{c}
D^{b b} \\
D^{b \zeta} \\
D^{\zeta \zeta}
\end{array}\right]=\nu_{12}(v)\left(\frac{v}{v_{\perp}}\right)^{3}\left(M_{1} v_{\perp}\right)^{2}\left[\begin{array}{c}
r^{2}\left(b_{p}^{2} I_{-}+\frac{1}{2} b_{t}^{2} I_{+}\right) \\
r R b_{p} b_{t}\left(I_{-}-\frac{1}{2} I_{+}\right) \\
R^{2}\left(b_{t}^{2} I_{-}+\frac{1}{2} b_{p}^{2} I_{+}\right)
\end{array}\right],
$$

with

$$
\nu_{12}(v) \equiv \frac{2 \pi e_{1}^{2} e_{2}^{2} \ln \Lambda n_{2}\left(r_{b 1}\right)}{M_{1}^{2} v^{3}}
$$

the collision frequency for species 1 on specjes 2 . For trapped particles, one has $\bar{v}_{\|}=0$, hence $a_{v}=\pi / 2$, so that $I_{ \pm}=1$. For deeply passing particles, $a_{v} \rightarrow 0$. In this limit, one has $I_{+} \rightarrow(4 / \pi) a_{v}, I_{-} \rightarrow(4 / 3 \pi) a_{v}^{3}$.

From Eqs.(51) and (21), we compute the radial diffusion coefficient $D^{r r} \equiv \boldsymbol{\epsilon}^{r} \cdot \mathbf{D} \cdot \boldsymbol{\epsilon}^{r}$ :

$$
D^{r r}= \begin{cases}\nu_{12} \rho_{g}^{2}\left[(q / \epsilon)^{2}+\frac{1}{2}\right] & (r=0), \\ \nu_{12} \rho_{g}^{2}\left(v / v_{\perp}\right)^{3}\left[\frac{1}{2} I_{+}+(\epsilon / q)^{2} I_{-}\right] & (r=1) .\end{cases}
$$


The first term in the $\tau=0$ expression here is the dominant, neoclassical term, yielding banana diffusion $D_{b m} \simeq \nu_{12} \rho_{g}^{2} q^{2} / \epsilon^{3 / 2}$ when averaged over pitch-angle. The second term for $\tau=0$, smoothly joining the first term for $\tau=1$ at the $\tau=0 \rightarrow 1$ transition, represents classical diffusion, $D_{d} \simeq \nu_{12} \rho_{g}^{2}$. The second term for $\tau=1$ is negligible, down from $D_{c l}$ by $(\varepsilon / q)^{2}$.

Diffusion in velocity space is also described by Eqs.(51). For example, for $\tau=1$, using Eqs.(23) and (51), one has

$$
\begin{aligned}
D^{\bar{v}_{\|} \bar{v}_{\|}} & \simeq \frac{1}{(q M R)^{2}} D^{J_{v} J_{v}}=\frac{1}{(q M R)^{2}}\left(D^{b 6}+2 q D^{6 \zeta}+q^{2} D^{\zeta \zeta}\right) \\
& =\nu_{12}(v) v_{\perp}^{2}\left(\frac{v}{v_{\perp}}\right)^{3} I_{\ldots} .
\end{aligned}
$$

Similarly, using Eqs.(46) and (51) along with $\partial J_{g} / \partial v_{\perp}=M v_{\perp} / \Omega_{g}$ and the fact that $\Omega_{\zeta} \simeq q \Omega_{b}$ for $r=1$, one finds

$$
\begin{aligned}
D^{v_{\perp} v_{\perp}} & =\left(\frac{\Omega_{g}}{M v_{\perp}}\right)^{2} D^{g g}=\left(\frac{\Omega_{b}}{M v_{\perp}}\right)^{2} D^{J_{v} J_{*}} \\
& =\nu_{12}(v) \bar{v}_{\|}^{2}\left(\frac{v}{v_{\perp}}\right)^{3} I_{-} .
\end{aligned}
$$

The factor $\left(v / v_{1}\right)^{3} I_{-}$appearing in Eqs. (53) and $(54)$ is a weak function of pitch angle, varying from 1 at $\alpha_{v}=\pi / 2$ to $\pi^{2} / 6$ at $\alpha_{v}=0$. For both $D^{\bar{v}_{\|} \bar{\nu}_{\|}}$ and $D^{v_{\perp} \boldsymbol{v}_{1}}$, one notes that only $I_{-}$, and not $I_{+}$, appears. This is because $\mathbf{D}$ is a particular $\mathbf{k}$-average $\langle\ldots\rangle_{\mathbf{k}}$ of $\bar{l} \bar{l}$, and so

$$
D^{J_{*} J_{\mathbf{V}}}=\left\langle\epsilon^{J_{v}} \cdot \overline{\mathbf{I}} \overline{\mathbf{I}} \cdot \epsilon^{J_{\bullet}}\right\rangle_{\mathbf{k}}=\left((m+n q)^{2}\right\rangle_{\mathbf{k}}=(q R)^{2}\left(k_{\|}^{2}\right\rangle_{\mathbf{k}} \propto I_{-},
$$

noting the definition of $I_{-}$in (50). For the turbulent spectrum, treated in the next section, one has $\left\langle k_{\|}^{2}\right\rangle_{\mathbf{k}} \simeq 0$. Thus, as concluded from earlier quasilinear calculations, ${ }^{6,1)}$ turbulence causes very little velocity-space diffusion ( $\left.\alpha I_{-}\right)$, even while quite effectively inducing radial transport.

We relate these results to the more familiar Lorentz collision operator. Parametrizing velocity-space by $\left(J_{g}, E^{\prime}\right)$, the bounce-averaged Lorentz operator may be written ${ }^{7}$

$$
C_{L} f=\Omega_{b} \partial_{J_{1}} \frac{2 \nu_{12}}{\Omega_{g}} J_{g} J_{v} \partial_{J_{q}} f
$$

This is to be identified with the velocity-space portion of the diffusive contribution in Eq. (1), written in the noncanonical variable set $y \equiv\left\{y^{i}\right\}$ in 
which $J_{b}$ in the canonical set $z \equiv(\theta, J)$ has been replaced by $E^{\prime}$. 'This diffusive term may be written

$$
\partial_{\mathbf{J}} \cdot \mathbf{D} \cdot \partial_{\mathbf{J}} f=\mathcal{J}^{-1} \partial_{\mathbf{y}^{\prime}} \mathcal{J} D^{y^{\prime} y^{\top}} \partial_{y^{\prime}} f
$$

where the Jacobian between the sets $y$ and $z$ is given by

$$
\mathcal{J} \equiv|\partial(z) / \partial(y)|=\left|\partial J_{b} / \partial E^{\prime}\right|=\left|\Omega_{b}^{-1}\right|
$$

Taking the restriction $C_{v} f$ of expression (56) to the $2 \times 2$ velocity sace submatrix of the full $\mathbf{D}$, and using the fact, following from Eq. (47), that $D^{E^{\prime} j}=D^{j E^{\prime}}=0$, one has

$$
\begin{aligned}
C_{v} f & =\Omega_{b} \partial_{J}, \Omega_{b}^{-1} D^{g g} \partial_{J} f \\
& =\Omega_{b} \partial_{J}\left[\frac{2 \nu_{12}}{\Omega_{g}} J_{g} q R M \bar{v}_{\|}\left(\frac{v}{v_{\perp}}\right)^{3} I_{-}\right] \partial_{J_{1}} f
\end{aligned}
$$

where we have used Eq. (54) and $\bar{v}_{11} \simeq q R \Omega_{b}$. One sees that Eqs. (55) and (57) are in agreement, making the identification $J_{v} \simeq q R M \bar{v}_{\|}\left(v / v_{\perp}\right)^{3} J_{-}$, in approximate agreement with (22).

\section{B. Turbulent Transport}

We now move to the somewhat longer wavelengths $\left(\lambda \sim \rho_{i} \gg \lambda_{D}\right)$ characterizing plasma microturbulence, and perform an analogous calculation of $D$, using the same expression (33) for the coupling coefficients used in the preceding subsection, but specialiced to this different regime.

Specifically, we consider the transport induced by magnetic microturbulence at vanishing collisionality. For thermal electrons, whose gyroradius and drifts across field lines may be neglected $\left(z_{g, b} \rightarrow 0\right)$, this mechanism was studied by Rechester and Rosenbluth, ${ }^{12}$ who found an anomalous diffusion coefficient for passing electrons

$$
D_{R R}^{r} \simeq \frac{\pi}{\left|\Omega_{b}\right|}(u \bar{b})^{2}
$$

where $\bar{b} \equiv \bar{B} / B$ is the average amplitude of the perturbing radial field $B_{\mathrm{r}}$, normalized to $B$. (Here, $u \equiv \overline{\left|y_{\mid}\right|}$is the bounce-average of the absolute value of the parallel velocity, nonzero for trapped as well as passing particles.) The modification of this mechanism by the $l$ rge $z_{g}$ and $z_{b}$ of energetic electrons 
and ions was estimated in Ref. 5 . Here, we do a more complete evaluation of $\mathbf{D}$ than was done there, for comparison with the collisional and ripple mechanisms considered in this section, and in preparation for the inclusion of the contribution from $\mathbf{F}$ in Sec.V.

We thus consider mides having $\phi$ and $A_{\perp}$ negligible, and therefore retain only the last two terms in Eq. (33). Additionally, the spectrum is characterized by $k_{\perp} \sim \rho_{i}^{-1}, k_{\|} \leq L_{a}^{-1}$, with $L$, the shear scale length. Thus, $k_{\perp} \gg k_{\|}$, as opposed to a typical contributing fluctuation for collisional transport, for which $k_{\perp} \sim k_{\|}$. For $A_{\perp}=0$, one has $\theta_{b A}=0$ in Eq. (33). Then, again using the stationary-phase approximation for the bounce-associated $J_{l}$ in Eq. (33), one finds

$h(1, J, \omega)=-\frac{e}{c} A \delta\left(l_{\zeta}-\pi\right) J_{l,} \sqrt{\frac{2}{\pi z_{b}}}\left(u_{1} \cos \theta_{z}-i u_{0} \sin \theta_{b+} \sin \theta_{z}\right) e^{-i\left(l_{b}-r m\right) \theta_{\partial+}}$

for $\left|l_{b}-\tau m\right|<z_{b}$, and

$$
h(1, J, \omega) \simeq 0
$$

for $\left|l_{b}-\tau m\right|>z_{b}$. Here, $\theta_{x} \equiv z_{b}-\left(l_{b}-\tau m\right) \pi / 2-\pi / 4$, and $u_{0}$, defined following Eq. (33), now simplifies to $u_{0} A=\Omega_{b}\left(\theta_{1} A_{\theta}+\zeta_{1} A_{\zeta}\right)$. From the definitions following Eq. (33), for $k_{\perp} \gg k_{\|}$, one has

$$
\sin \theta_{b k} \equiv-k_{r} r_{1} / z_{b} \simeq-k_{r} / k_{\perp} \text {, }
$$

and

$$
u \simeq \begin{cases}(2 / \pi) u_{0}, & (\tau=0) \\ \left|u_{1}\right|, & (\tau=1),\end{cases}
$$

with Eqs. (62) valid not too near the trapped/passing boundary.

As in subsection $A$, one may replace the contributions $\cos ^{2} \theta_{2}$ or $\sin ^{2} \theta_{2}$ in $|h|^{2}$, oscillatory in $b_{b}$, by their pairwise average. Thus, again using Eq. (42), one can write

$$
|h(1, J, \omega \mid a)|^{2} \simeq|\bar{h}(\mathbf{J} \mid a)|^{2} \delta\left(l_{\zeta}-n\right) J_{l_{\mathrm{l}}}^{2} \overline{J_{l_{0}-\tau_{m}}^{2}},
$$

where $|\bar{h}(\mathbf{J} \mid \boldsymbol{c})|^{2}=\left|(e / c) u_{\tau} A\right|^{2}$, with

$$
u_{\tau} \equiv \begin{cases}u_{0} \sin \theta_{b k}, & (\tau=0) \\ u_{1}, & (\tau=1)\end{cases}
$$


Using (63), we write $|\alpha|^{2}$ in a form paralleling the symmetric collisional expression (37):

$$
\begin{aligned}
& |\alpha|^{2}=\left.i \bar{\alpha}\right|^{2} \delta\left(l_{1 \zeta}-n\right) \delta\left(l_{2 \zeta}-n\right) J_{l_{1}},{ }^{2} \overline{J_{l_{b}}-r_{1} m^{2}} J_{l_{2}}{ }^{2} \overline{J_{l_{2 b}-r_{2} m}{ }^{2}} \\
& |\bar{\alpha}|^{2}=:\left.\overline{\bar{\alpha}}\right|^{2} s\left(w_{a} / 2, r_{b 1}-r_{a}\right) s\left(w_{a} / 2, r_{b 2}-r_{a}\right), \\
& |\overline{\bar{\alpha}}|^{2}=\left|\frac{\overline{\bar{h}}(1 \mid a) \overline{\bar{h}}^{*}(2 \mid a)}{N_{a} \Delta_{a}}\right|^{2},
\end{aligned}
$$

where we have written ${ }_{1} \bar{h}_{1}{ }^{2}$ with its localizing factor $s()$ explicitly displayed,

$$
\left.\bar{h}\right|^{2}=[\bar{h})^{2} s\left(w_{a} / 2, r_{b}-r_{a}\right) .
$$

Summing over $\mathrm{l}_{2}$ as done in Eq. (38), one finds

$$
D(1 \mid 2)=\sum_{a} \sum_{l_{1,}, l_{1+}} l_{1} l_{1} \pi \delta\left(l_{1} \cdot \Omega_{1}^{\prime}\right)\left|\bar{h}_{t h}(1 \mid a, 2)\right|^{2} J_{l_{1}}{ }^{2} \overline{J_{l_{1 b}-n m^{2}}}
$$

the same as yielded by the quasilinear expression (7), with thermal spectrum

$$
\begin{aligned}
\mid \bar{h}_{t h}(1 \mid a, 2) !^{2} & \equiv \int d^{6} z_{2} f(2)|4 \pi \bar{\alpha}|^{2} \\
& =\left.! \bar{h}(1 a)\right|^{2} \int d^{6} z_{2} f(2)\left|\frac{4 \pi \bar{h}(2 \mid a)}{N_{a} \Delta_{a}}\right|^{2} .
\end{aligned}
$$

The arguments $(1 \mid a, 2)$ here mean "the spectrum felt by species 1 from that portion of fluctuations $a$ which are driven by species 2."

As discussed in the Introduction, the thermal fuctuations given by Eq. (66) do not properly represent the turbulent spectrum of realistic experiments. Thus, we replace $\left|\bar{h}_{t h}\right|^{2}$ in Eq. (65) by a model spectrum, $|\bar{h}(1 \mid a, 2)|^{2}$, satisfying the general characteristics of the turbulent spectrum given earlier. We assume that $\Delta_{a}$ in expression (66) is nonlinearly modified from its thermal value, so that the fields $A(a, 2)$ driven by species 2 , given by the $z_{2}$ integration in (66), are given by

$$
\begin{aligned}
& |A(a, 2)|^{2} \equiv|\overline{\bar{A}}(a, 2)|^{2} s\left(w_{a} / 2, r_{b 1}-r_{a}\right), \\
& |\bar{A}(a, 2)|^{2}=\frac{V_{A}}{V_{a}} \frac{(2 \pi)^{3 / 2} \bar{B}^{2}}{\left(\Delta k_{\perp}\right)^{2}} \exp \left[-\frac{k_{\perp}^{2}}{2\left(\Delta k_{\perp}\right)^{2}}-\frac{k_{\|}^{2}}{2\left(\Delta k_{\|}\right)^{2}}\right] .
\end{aligned}
$$

Here, $\Delta k_{\perp} \sim \rho_{i}^{-1}$ and $\Delta k_{\|} \sim L_{a}^{-1}$ are the spectrum widths in the perpendicular and parallel directions, $V_{A}^{-1} \equiv\left(\Delta k_{\mathfrak{L}}\right)^{2} \Delta k_{\|}$measures the volume 
in $\mathbf{k}$-space over which $A^{2}(\mathbf{k})$ is apprecjable, and $\tilde{B}^{2}$ measures the overall strength of the turbulent fluctuations. The normalization is chosen so that

$$
\left\langle B_{r}^{2}\right\rangle \equiv V_{p}^{-1} \int d \mathbf{x}\left|k_{Q} \cdot A(\mathbf{x})\right|^{2}=\bar{B}^{2} .
$$

Thus, $|\bar{h}(1 \mid a, 2)|^{2}$ has the same form as $|\bar{h}(1 \mid a)|^{2}$ in Eq. (63), but with $\mid A^{2}$ there replaced by $|A(a, 2)|^{2}$ in Eq. (67). [More detailed use will be made of the structure of the spectrum in Eq. (66) in Sec. V, where we consider the relative contributions to the spectrum from different species.]

Because $k_{\|} \ll k_{\perp},\left(m \theta_{1}+n \zeta_{1}\right)$ in $z_{b}$ is given by the perpendicular drift motion. Within a flux surface, $\left(m \theta_{1}+n \zeta_{1}\right) \simeq k_{q} q_{1} \simeq k_{q} r_{1}$, and thus,

$$
z_{b} \simeq k_{-} r_{1} \simeq k_{\perp} v_{B} / \Omega_{b} \text {. }
$$

The summation over the $\left(\omega_{g}, \omega_{b}\right)$ or $\left(l_{b}, l_{b}\right)$-plane in Fig. 1 is conceptually the same as for collisional transport, but, because the wavelengths involved are longer, the characteristic frequencies are smaller. Two consequences of the fact that $k_{\|} \ll k_{-}$are first, that the resonance line in Fig. 1 passes through the contributing rectangle very nearly centered about $l_{g}=0$, and second, the height $\Delta \omega_{b}$ of the contributing rectangle about $\omega_{b}=\tau m \Omega_{b}$ is so small that the resonance line crosses only a very few values of $l_{g}$. From Eq. (41), the change $\delta l_{g}$ in $l_{g}$ of the resonance line in crossing the rectangle is

$$
\delta l_{g} / \pi=\Delta \omega_{b} / \Omega_{g} \simeq \epsilon^{\frac{1}{2}} k_{\|} \rho_{g}+\left(\rho_{g} / 2 R\right) z_{g} .
$$

For the turbulent spectrum, the term in $k_{\|}$in (69) (which dominated for a typical mode of Sec.A) is totally negligible. The term in $z_{g}$ is negligible as well, except for extremely energetic ions, such as alpha particles. Even for these (using TFTR-like parameters $T_{i} \simeq 10 \mathrm{keV}, R=2.5 \mathrm{~m}, B=5 \mathrm{~T}$ ), one has $\rho_{g} \simeq 5.2 \mathrm{~cm}, z_{g} \equiv k_{\perp} \rho_{g} \sim \rho_{g} / \rho_{g i} \simeq 26$, hence $\delta l_{g} \sim 0.8$. Therefore, for most particles, only the $l_{a}=0$ term contributes, while for alphas, the $l_{g}= \pm 1$ terms may or may not also contribute, depending upon the specifics of the turbulence.

In the absence of $l_{g} \neq 0$ contributions, one has $\dot{\mu}=\dot{J}_{g}=0, D^{g j}=D^{i g}=$ 0 , and $F^{\theta} \equiv \epsilon^{g} \cdot \mathbf{F}=0$, i.e., no pitch-angle scattering can occur. Thus, the turbulent contribution to pitch-angle scattering of these $l_{g} \neq 0$ terms might be of interest for very energetic ions. $A$ similar problem has been treated by Putvinskii and Shurygin ${ }^{13}$ considering TF-ripple as the perturbation, but the wavelength from this source is too long to produce significant effects. ${ }^{14}$ 
Here, we treat the case where only the $l_{g}=0$ harmonic contributes, which applies to most classes of particles and types of turbulence.

Whether a single or several terms are kept, the sum over $l_{g}$ in (65) may not be converted into an integration, in contrast to the case of collisional transport. However, since the factor $J_{l g}{ }^{2}$ in Eq. (65) is rapidly oscillatory in the particle energy and pitch-angle, one may replace it with the averaged form (42) as well. Making this replacement, and the replacement $\mid h_{t h}{ }^{2} \rightarrow$ $|h|^{2}$ just discussed, from (65) we write the analogue of expression (43) :

$$
\begin{aligned}
D^{i j}(1 \mid 2)= & \int \frac{d \mathbf{k}}{(2 \pi)^{3}} V_{a}|\bar{h}(1 \mid \mathbf{k} .2)|^{2} \int_{-\infty}^{\infty} d_{\omega_{b}} \Omega_{\eta} \sum_{l_{p}} l_{i} l_{j} \pi \delta\left(1 \cdot \mathbf{\Omega}_{l}^{\prime}\right) \times \\
& \frac{s\left(\Delta \omega_{b} / 2, \omega_{b}^{\prime}\right)}{\Delta \omega_{b}} \frac{s\left(\Delta \omega_{g} / 2, \omega_{g}\right)}{\Delta \omega_{g}} \\
= & \left.\pi \int \frac{d k}{(2 \pi)^{3}} V_{a}|\bar{h}(1 \mid k, 2)|^{2} \Omega_{g} \sum_{l,} l_{i} l_{j} \frac{s\left(\Delta \omega_{b} / 2, \omega_{b 0}^{\prime}\right)}{\Delta \omega_{b}} \frac{s\left(\Delta \omega_{g} / 2, \omega_{g}\right)}{\Delta \omega_{g}}\right|_{1=1},
\end{aligned}
$$

where

$$
\omega_{b 0}^{\prime}\left(l_{g}, m, n\right) \equiv-\left(\omega_{g}+n \Omega_{\zeta}^{\prime}+\tau m \Omega_{b}\right)= \begin{cases}-\left(l_{g} \Omega_{g}+n \Omega_{\zeta}^{\prime}\right), & (\tau=0) \\ -\left(l_{g} \Omega_{g}+k_{\|} \bar{v}_{\|}\right), & (\tau=1)\end{cases}
$$

and $\bar{I}$ is as given in Eq. (45). (Because $\Delta \omega_{b} \ll \Delta w_{g}$, the "short and broad" approximation used in Eq. (44) is still better satisfied here.)

Now, we impose the additional relation that $\vec{l}_{g}=0$. Thus, Eqs. (46), while still true, are replaced by the si pler relations already mentioned,

$$
D^{9 j}=D^{i g}=0 \text {. }
$$

Since $k_{\| \mid} \ll k_{\perp}$, we neglect $k_{\| \mid}$in $m$ and $n$, hence $m \simeq r b_{t} k_{q}, n \simeq-R b_{p} k_{q}$, and thus

$$
D^{b b}=-q D^{b \zeta}=-q D^{\zeta b}=q^{2} D^{\zeta \zeta,}
$$

leaving only a single component $D^{i j}$, which we take as $D^{\varangle 6}$, to be determined.

Both $\omega_{b 0}^{\prime}$ and $\omega_{g}$ in the s-functions in (70) are approximately zero, so both of these may be replaced by unity. Keeping only the $t_{g}=0$ term there, using (67) in (70), the reraaining $k$-integrals needed are again easily evaluated:

$$
\int \frac{d k}{(2 \pi)^{3 / 2}} \frac{\exp \left[-k_{\perp}^{2} / 2\left(\Delta k_{\perp}\right)^{2}-k_{\| 1}^{2} / 2\left(\Delta k_{\| 1}\right)^{2}\right]}{\left(\Delta k_{\perp}\right)^{2} \Delta k_{i}} \frac{k_{q}^{2}}{k_{\perp}^{2}}\left[\begin{array}{c}
\sin ^{2} \theta_{3 k} \\
1
\end{array}\right]=\left[\begin{array}{l}
1 / 8 \\
1 / 2
\end{array}\right],
$$


where we have used Eq. (61). The upper (lower) component here is needed for $T=0(1)$. Using Eqs.(74), one finds

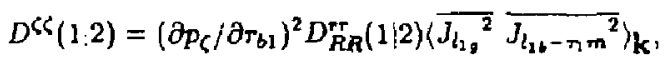

with $\partial p_{c} / \partial r_{b} \equiv-\left(M \Omega_{g} R b_{p}\right)=-\left(e B R b_{p} / c\right) . D_{R R}^{r F}(1 ; 2)$ is as given in $(58)$, with $u=u(1), \dot{b}$ due to species 2 [via Eq.(67)], and

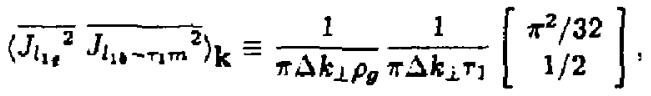

where the upper (lower) component again applies for $\tau=0(1)$.

Parallel to Eqs. (52)-(54) of the preceding section, from Eqs. (21) and (23), one has

$$
D^{r r}=D_{R R}^{r r}\left\langle\overline{J_{l_{1 q}{ }^{2}}} \overline{J_{l_{1 b}-\tau_{1} m^{2}}}\right\rangle_{\mathbf{k}}
$$

for $\tau=0$ and 1 , and

$$
D^{\text {uu }} \propto D^{v_{-} v_{\perp}} \propto D^{J_{\mathbf{g}} J_{\mathbf{r}}} \propto\left\langle k_{\|}^{2}\right\rangle_{\mathbf{k}} \simeq \mathbf{0}
$$

The reduction of the Rechester-Rosenbluth result due to finite $z_{\mathrm{g}}$ and $z_{b}$, discussed in Ref. 5 , is contained in the factor $\langle. .\rangle_{k}$ in Eqs. (75) and (77). As discussed in more general terms around Eq. (36), this factor measures the (square of) the fraction of each gyro- and bounce- time that a particle spends locally resonant with a given perturbing mode $a$. In the $z_{g, b} \rightarrow 0$ limit, where this fraction becomes unity (or zero), the $J_{l}^{2}$ there become Kronecker $\delta$-functions $\delta(l)$, and the Rechester-Rosenbluth result is recovered.

\section{Ripple Transport (Stochastic Regime)}

We complete the series of illustrations for this section by computing from the same expressions for $h(\mathbf{l}, \mathbf{J}, \omega)$ and $\mathbf{D}$ a ripple-transport result. Specifically, we generalize the expression for the "stochastic regime"l5 to include an electrostatic component, and to ripple perturbations having $m \neq$ $0, \omega \neq 0$, and to allow $q N \equiv q n+m$ of order unity, as well as the limit $q N \gg 1$ previously assumed. These generalizations make the theory applicable to internally generated modes, including low-n MHD-modes, as well as to the TF- coil ripple with which the theory was originally concemed. This is the same generalization for the stochastic regime which Ref. $?$ achieved for the more collisional "banana drift" regimes, and is thus relevant for very energetic jons, such as alpha particles. 
The modes we consider have still longer wavelength than those of the previous subsections, long enough so that $\delta l_{g}$ discussed around Eq. (69) is small compared with unity (even for alphas). Then only terms $I$ with $l_{g}=0$ contribute to $\mathrm{D}$, so that Eq.(72), which held for almost all particles in turbulence, holds for all particles here. Thus $J_{g}$ is constant, and a guidingcenter description of the particle motion is valjd.

It has been noted that the second term in Eq. (33) yields the $\mu B_{1}$ perturbation in the guiding-center Hamiltonian $H_{G}$. Taking the small- $z_{g}$ limit (34) of the $J_{l}$, 's there, and setting $l_{g}=0$, this second term reduces to (momentarily restoring mode index $\mathbf{k}$ for clarity)

$$
\begin{aligned}
& h(\mathbf{l}, \mathbf{J}, \omega a)=-\frac{1}{2} \frac{e}{c} v_{-} \rho_{g} i k_{\perp} A_{-} \mathbf{k}^{\sin \left(\theta_{g A}-\theta_{g k}\right) \delta\left(l_{\zeta}-n\right) J_{l_{b}-m} \times} \\
& e^{-i\left(l_{b}-\tau m\right) \theta_{\Delta, 1}-i l_{g} \theta_{0} k} \\
& =\left(\mu B_{1 \mathbf{k}}\right) \delta\left(l_{\zeta}-n\right) J_{l_{\mathbf{b}}-\tau m} e^{-i\left(l_{\mathbf{b}}-\tau m\right) \theta_{\mathbf{k}}-i l_{\mathbf{l}} \theta_{\mathbf{3}} k},
\end{aligned}
$$

where $B_{j \mathbf{k}} \equiv \hat{b} \cdot \mathbf{i k} \times \mathbf{A}_{\mathbf{k}} \sin \left(\theta_{g A}-\theta_{g k}\right)$ is the perturbation to $B \equiv|\mathbf{B}|$ due to $A_{k}$. A more fundamental derivation of this form, which does not rely on the eikonal representation (24), comes from recognizing that the $l_{g}=0$ Fourier component of $h(\mathbf{l}, \mathbf{J}, \omega)$ simply involves a line-integration around a gyro-orbit:

$$
\begin{aligned}
\oint \frac{d \theta_{g}}{2 \pi} h(\theta, J) & =-\frac{e \Omega_{g}}{2 \pi c} \oint \boldsymbol{n}_{\perp} \cdot \mathbf{A}\left(\mathbf{R}+\rho_{g}\right) \\
& =\frac{e \Omega_{g}}{2 \pi c} \oint d \mathbf{S}_{\mid} \cdot(\nabla \times \mathbf{A})=\frac{e \Omega_{g}}{2 \pi c}\left(\pi \rho_{g}^{2}\right) B_{1}=\mu B_{1},
\end{aligned}
$$

where $d l_{\perp} \equiv v_{\perp} d t$ is an incremental line element, and $d \mathbf{S}_{\|} \equiv \dot{b} d S_{\|}$is an element of area on the disk formed by the gyro-orbit. Expression (79) has the same form as the first term in (33), to which it may be added to yield the full coupling coefficient for the ripple problem,

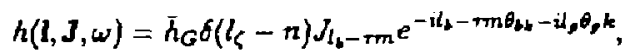

where $\bar{h}_{G} \equiv \epsilon \phi\left(r_{b}, m, n\right)+\mu B_{1}\left(r_{b}, m, n\right)$ is the amplitude of the perturbing portion of $H_{G}$, from which ripple-transport calculations normally begin.

As usual in ripple calculations, we consider the effects of a single mode a (or k). This perturbation may be internally or externally induced. For either, the quasilinear expression (7) applies. Thus, using Eq.(81) in (7), one has (suppressing species subscript " 1 " for simplicity of notation)

$$
\mathbf{D}(1)=\left.\sum_{l_{b}} 11 \delta\left(1 \cdot \Omega_{1}-\omega\right) \vec{h}_{G}^{2} J_{l-\pi m}{ }^{2}\right|_{l_{c}=\pi}
$$


This parallels Eq. (38) or (65) of the previous sections, except that it lacks the sums over both $l_{g}$ and mode index $a$, and so is substantially simpler to evaluate. Diffusion now occurs only due to overlap of the resonances of successive bounce-harmonics $l_{b}$, equivalent to the overlap criterion given in Ref. 15. When overlap exists, the sum over $l_{b}$ may be converted to an integral, as in the previous subsections, yielding

$$
D^{i j}(1)=\frac{\pi}{\left|\Omega_{b}\right|} l_{i} l_{j} \bar{h}_{G}^{2} \overline{J_{l_{b}-T m}}\left(z_{b}\right)_{\mid=I}
$$

where now

$$
\overline{\mathrm{j}}=\left(0,\left(\omega-\pi \Omega_{\zeta}\right) / \Omega_{b}, n\right) .
$$

Thus, Eqs. (72) again hold, as already noted. Specializing to $\tau=0$ for comparison with the previous theory, from Eqs. (83) and (21) one finds

$$
D^{r r}=\left.\frac{\pi}{4\left|\Omega_{b}\right|} i^{2} \overline{J_{l_{b}}{ }^{2}}\left(z_{b}\right)\right|_{l_{b}}
$$

Here, $\dot{v} \equiv 2 q n \bar{h}_{G} /\left(M \Omega_{g} r\right)$ is the amplitude of the radial drift $\dot{r}_{b}$ due to the ripple, given by

$$
\dot{r}_{b}=\boldsymbol{\epsilon}^{r} \cdot \dot{\mathbf{J}}=-\dot{v} \sum_{l_{b}} J_{l_{b}} \sin \left(n \zeta_{0}+l_{b} \theta_{b}-\omega t\right),
$$

following from Eq. (5). The physics of the result (84) may be described as follows almost the same description may be applied to the turbulent result (77)]: a particle resonant with bounce harmonic $l_{b}$ performs a random walk, taking a radial step at velocity $\hat{v} J_{b}$, for coherence time $\tau_{b}$. The factor $J_{b}$ represents the fraction of the full bounce period when the radial motion is non-oscillatory. Contrary to the usual lore, one notes that the point on the bounce-orbit during which this radial step is taken is not at the particle's turning points, in general. From Eq. (36), the step is taken at the turning point only for $\bar{l}_{b} / z_{b} \simeq 0$, a condition holding only for particles precessing at atmost the same frequency as the ripple perturbation (i.e., $w \simeq n \Omega_{\zeta}$ ). This condition does not hold, for example, for a typical alpha particle precessing in ripple due to TF coils, for which $\bar{l}_{b} / z_{b}$ can be comparable to unity. One also notes the dependence of $\bar{l}_{b}$ on mode frequency $w$. For $\left(w-n \Omega_{6}\right)$ large enough, the factor $\overline{J_{b}{ }^{2}}$ can be made to move into the $\tilde{l}_{b}>z_{b}$ limit of the Bessel function, where $\overline{J_{b}{ }^{2}}$, and so $D^{\text {rr }}$, fall off rapidly.

For the longer-wavelength modes of this section, one has $z_{b} \simeq m \theta_{1}+$ $n \zeta_{1} \simeq q N \theta_{1}$. One recovers the result of Ref. 15 by letting $\bar{h}_{G}=\mu B_{1}, \omega=0=$ 
$m$, and assuming $z_{b} \simeq q n \gg 1$, so that the large- $z$ form in (42) may be used for $\overline{J_{b}{ }^{2}}$. For $z_{b} \sim 1$, the point on a bounce orbit where the particle receives a radial kick is no longer localized, and correspondingly, the stationary-phase form (42) fails. Then the full form of the Bessel function must be retained.

\section{Evaluation of $\Gamma$}

Having gained experience through the evaluation of $\mathbf{D}$ with much of the mathematical mechanics, we can proceed to consider the effect of reinstating self-consistency, by computing the full flux $\Gamma$ in $J$-space, retaining both the $\mathbf{D}$ and $\mathbf{F}$-contributions.

We will perform this evaluation for the same turbulent mechanism as examined in Sec. IV-B, because this yields results which are new, and which can thus be compared with the analogous results for collisional symmetric transport, which are already well-established.

Up to this point, nothing has been said about the specific form of the distribution functions $f(J)$. We now adopt the near-equilibrium form for both species (suppressing species label)

$$
f_{0}(\mathbf{J}) \equiv \frac{\pi}{(2 \pi T)^{3 / 2}} \exp \left(-K_{0} / T\right),
$$

where $n, \Phi$, and $T$ are functions of $r_{b}(\mathbf{J}), K_{0} \equiv H_{0}-\epsilon \Phi$ is the (unperturbed) kinetic energy when a particle is at $r=r_{b}$, and for simplicity we take equal temperature distributions, $T_{1}=T_{2}=T$. If $r_{b}$ in (86) were replaced by $r$, $f_{0}$ would be of the "local Maxwellian" form $f_{M}$ used as the lowest-order distribution function in more standard approaches to transport. Because it is a function of $J$ alone, $f_{0}$ is an exact solution of the unperturbed ( $\left.h=0\right)$ Liouville equation, and thus contains, in addition to $f_{M}$, the collisionless correction (in banana width to minor radius) to $f_{M}$, which is what produces the radial fluxes. ${ }^{10,16}$ We thus substitute Eq. (86) into expression (15) for $\Gamma(1 \mid 2)$ to compute the transport.

To evaluate $\Gamma(1 \mid 2)$, one needs $1 \cdot \partial_{\mathbf{J}} f$. Using $f_{0}$ in (86) for $f$, one finds

$$
\mathbf{1} \cdot \partial_{\mathbf{J}} f_{0}=\left(\mathbf{1} \cdot \boldsymbol{\epsilon}^{\mathrm{r}} \boldsymbol{A}-\mathbf{1} \cdot \mathbf{\Omega} / T\right) f_{0}
$$

where

$$
A \equiv A_{n K}+\frac{e \Phi^{\prime}}{T}, A_{n K} \equiv A_{n}+A_{K} \frac{K_{0}}{T}, A_{n} \equiv\left(\frac{n^{\prime}}{n}-\frac{3}{2} A_{K}\right), A_{K} \equiv \frac{T^{\prime}}{T}
$$


describes the thermal forces. 'The prime denotes derivative with respect to $r_{b}$.) Lsing (64) and (87) in Eq. (15), one finds

$$
\begin{aligned}
& -\Gamma(12)=\int d^{6} z_{2} \sum_{\mathbf{a}} \sum_{\mathbf{l}_{1}, \mathbf{l}_{2}} \pi \delta\left(\Omega_{\text {res }}\right) \mid 4 \pi \alpha !^{2} \mathbf{I}_{1}\left(\left(l_{1} \cdot \partial_{\mathbf{J}_{1}}-\mathbf{l}_{2} \cdot \partial_{\mathbf{J}_{2}}\right)\right) f_{0}(1) f_{0}(2)
\end{aligned}
$$

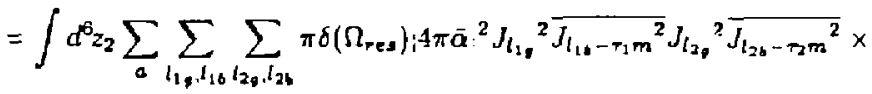

$$
\begin{aligned}
& \mathrm{l}_{1}\left(\mathrm{l}_{1} \cdot \epsilon^{r_{2}} A_{1}-\mathrm{l}_{2} \cdot \epsilon^{r_{2}} \cdot A_{2}\right) f_{0}(1) f_{0}(2) \text {, }
\end{aligned}
$$

where the contributions from $D\left(\sim l_{1} l_{1}\right)$ and $F\left(\sim l_{1} l_{2}\right)$ are still apparent The contributions to these terms from the term in $1 . \Omega$ in (87) have cancelled, due to the argument $1_{1} \cdot \boldsymbol{\Omega}_{1}-1_{2} \cdot \boldsymbol{\Omega}_{2}$ of the $\delta$-function. (An analogous cancellation occurs in linearizing the standard BL-operator.)

One notes in Eq. (89) the same $z_{2}$-integral over $|4 \pi \bar{\alpha}|^{2}$ which produced the scattering spectrum $\left.\bar{h}(1 ; a, 2)\right|^{2}$ in the calculation in Sec.IV $\cdot \mathbf{B}$ of $\mathbf{D}(1 ; 2)$, and the same identification can be made here in computing $\Gamma(1 ; 2)$. Here, however, we wish to consider the symmetries which the retention of $\mathbf{F}$ creates between $\Gamma(1,2)$ and $\Gamma(2 ; 1)$, and this requires that we make further use of the spectral structure given in Eq. (66), to strip away the $z_{2}$-integration present in the model spectrum (67). Dropping the subscript "th" on $\bar{h}_{\text {th }}$ in Eq. (66), an expression for $|\bar{a}|^{2}$ yjelding the spectrum (67) is

$$
|4 \pi \bar{\alpha}(1,2 \mid a)|^{2}=\left.\frac{4 \pi e_{1} e_{2}}{N_{a} \Delta_{a}} \frac{u_{r}(1)}{c} \frac{u_{\tau}(2)}{c}\right|^{2},
$$

with $s_{a}$ given by

$$
\left|\frac{4 \pi e_{1} e_{2}}{\left.N_{a}\right\lrcorner_{a}}\right|^{2} \equiv\left|e_{1} \overline{\bar{A}}(a, 2)\right|^{2} /\left[V_{\mathrm{a}} n_{2}\left(\mid u_{-}(2) / c_{i}^{2}\right) j .\right.
$$

Here, $V_{a} n_{2}\langle\ldots\rangle \equiv \int_{V_{a}} d^{6} z_{2} f(2)(\ldots)$, where $\int_{V_{0}} d^{6} z_{2}(\ldots) \equiv \int d^{6} z_{2} s\left(., r_{b 2}-\right.$ $\left.r_{a}\right)(\ldots)$ is the phase space integral over the toroidal shell $V_{a}$. Thus, the desired model spectrum $|\bar{A}|^{2}$ is achieved in Eq. (91) by modifying the dielectric function $\Delta_{a}$ from its form in a stable plasma, which would produce a thermal spectrum.

As in Sec. IV, we simplify the summarion over $l_{2}$ by taking the Lorentz limit $\mathbf{l}_{\mathbf{2}} \cdot \boldsymbol{\Omega}_{\mathbf{2}} \rightarrow n \boldsymbol{\Omega}_{\zeta E}$, and then approximately perform the summation over $l_{1}$, again using (42). Equation (89) then yields

$$
-\Gamma(1: 2)=\int d^{3} z_{2} \sum_{a} \bar{Q}(1,2 \mid a) \overline{\mathbf{l}}_{1}\left(\overline{\mathbf{I}}_{1} \cdot \epsilon^{r_{1}} A_{1}-\overline{\mathbf{I}}_{2} \cdot \epsilon^{r_{2}} A_{2}\right) f_{0}(1) f_{0}(2),
$$


with the 1-averaged kernel $\bar{Q}$ given by

$$
\begin{aligned}
& \bar{Q}(1,2 a)=\bar{Q}(1,2 a) s\left(,, r_{b 1}-r_{a}\right) s\left(., r_{b 2}-r_{a}\right), \\
& \bar{Q}(1,2 a) \equiv \pi \Omega_{g 1} \frac{s\left(\Delta \omega_{g 1} / 2, \omega_{g}\right)}{\Delta \omega_{g 1}} \frac{s\left(\Delta \omega_{b 1} / 2, \omega_{b 0}^{\prime}\right)}{\Delta \omega_{b 1}} ; 4 \pi \overline{\bar{\alpha}}^{2} \mathbf{z}_{1=1} \\
& =\pi \Omega_{g 1} \frac{s\left(\Delta \omega_{g 1} / 2, \omega_{g}\right)}{\Delta_{\omega_{g 1}}} \frac{s\left(\Delta \omega_{b 1} / 2, \omega_{b 0}^{r}\right)}{\Delta \omega_{b 1}} \frac{\left|u_{\tau}(1)\right|^{7}\left|\frac{c_{1}}{c} \overline{\bar{A}}(a, 2)\right|^{\prime} u_{\tau}(2) i^{2}}{\zeta_{a} n_{2}\left(\left|u_{\tau}(2)^{2}\right\rangle\right.} \text { I. }
\end{aligned}
$$

Here, as in Sec. IV-B, we have assumed that only $l_{1 g}=0$ contributes, so that $\bar{l}_{1,2}$ are again given by $\bar{l}=(0, \tau m, n)$. With $\mathrm{Eq}$.(93), one readily sees that the term in $\bar{l}_{1} \bar{l}_{1}$ in Eq. (92) yields expression (70), as it should.

From Eq. (21), one has

$$
e \epsilon^{r} \cdot \bar{l}=\left(-c / B R b_{\mathbf{p}}\right) n
$$

for any species, and for $\tau=0,1$. Therefore, the terms in $e \Phi^{\prime}$ in the thermal force factor in Eq. (92) cancel, resulting in property (3) of turbulent (or symmetric) transport noted in Sec. I:

$$
\begin{aligned}
\left(\bar{l}_{1} \cdot \epsilon^{r} \cdot A_{1}-\bar{l}_{2} \cdot \epsilon^{r \cdot} \cdot A_{2}\right) & =n\left(\frac{-c}{B R b_{p}}\right)\left(\frac{1}{e_{1}} A_{1}-\frac{1}{e_{2}} A_{2}\right) \\
& =n\left(\frac{-c}{B R b_{p}}\right)\left(\frac{1}{e_{1}} A_{n K 1}-\frac{1}{e_{2}} \cdot A_{n K 2}\right) .
\end{aligned}
$$

The $\mathbf{k}$-integration over $\overline{\bar{Q}}$ in (92) yields the same $D^{\varsigma(1 / 2)}$ as in Eq. (70) or (75) :

$$
\begin{aligned}
\sum_{\mathbf{k}} \bar{Q}(1,2 \mid a) n^{2} & =\frac{\pi}{\left|\Omega_{b 1}\right|} \sum_{\mathbf{k}}|\overline{\bar{h}}(1 \mid, \mathbf{k}, 2)|^{2} \overline{J_{l_{1}}{ }^{2}} \frac{J_{l_{14}-r_{1} m^{2}} n^{2}}{V_{a} n_{2}\left\langle\left|u_{7}(2)\right|^{2}\right\rangle} \\
& =D^{\zeta(1 \mid 2)} \frac{\left|u_{-}(2)\right|^{2}}{V_{a} n_{2}\left(\left|u_{-}(2)\right|^{2}\right\rangle}
\end{aligned}
$$

Using this in Eq. (92), one finds

$$
-\Gamma(1: 2)=\left.\left(\frac{-c}{B R b_{p}}\right) D^{\zeta 6}(1 \mid 2)\left(\frac{1}{e_{1}} A_{1}-\frac{1}{e_{2}} A_{2}\right)\left[\begin{array}{c}
0 \\
-g \tau_{1} \\
1
\end{array}\right] f_{0}(1)\right|_{r_{b 1}=r_{12}}
$$


Dotting this with $\epsilon^{r_{1}}$, the radial flux is given by

$$
-\Gamma^{r}(12) \equiv-\epsilon^{r_{2}} \cdot \Gamma(12)=e_{1} D^{r r}(1 ; 2)\left(\frac{1}{e_{1}} A_{1}-\frac{1}{e_{2}} A_{2}\right) f_{0}(1)_{r_{b 1} \simeq r_{b 2}} .
$$

We now consider $\Gamma(21)$. This is given by interchanging species indices $l$ and 2 in Eq. (89). We evaluate the resulting expression in the same limit $1_{2} \cdot \Omega_{2}-n \Omega_{\zeta E}$ used with $\Gamma(1 \mid 2)$. This yields

$$
-\Gamma(2 I)=\int a^{\beta} z_{1} \sum_{a} \bar{Q}(1,2 \mid a) 1_{2}\left(1_{2} \cdot \epsilon^{r_{2}} A_{2}-1_{1} \cdot \epsilon^{r} \cdot A_{1}\right) f_{0}(1) f_{0}(2),
$$

with $\bar{Q}$ the same as given in Eq. (93). Except for the integration over $z_{1}$ instead of $z_{2}$, the evaluation of this is essentially the same as for $\Gamma(1 \mid 2)$ in Eq. (92). With (96), one has

$$
\begin{aligned}
-\Gamma(2: 1)= & \left.\int_{r_{a}^{r}} d^{6} z_{1} f_{0}(1)\left(\frac{-c}{B R b_{p}}\right) D^{\zeta \zeta}(1 ! 2)\right] \frac{\left|u_{\tau}(2)\right|^{2}}{V_{0} n_{2}\left\langle\left(u_{\tau}(2) ; ;^{2}\right\rangle\right.} \times \\
& \left.\left(\frac{1}{e_{2}} A_{2}-\frac{1}{e_{1}} A_{1}\right)\left[\begin{array}{c}
0 \\
-q \tau_{2} \\
1
\end{array}\right] f_{0}(2)\right|_{r_{b 2}=r_{b 1},}
\end{aligned}
$$

and thus

$$
-\Gamma^{r}(2 ! 1) \equiv-\epsilon^{r 3} \cdot \Gamma(2 \mid 1)=\left.e_{2} D^{r r}(2.1)\left(\frac{1}{e_{2}} \cdot A_{2}-\frac{1}{e_{1}} A_{1}\right) f_{0}(2)\right|_{r_{82}} \simeq r_{b 1}
$$

where

$$
e_{2}^{2} D^{r r}(2 \mid 1) \equiv \int_{v_{a}} d^{6} z_{1} f_{a}(1) e_{1}^{2} D^{r r}(12) \frac{\left.u_{r}(2)\right|^{2}}{V_{a}^{r} n_{2}\left\langle\left|u_{T}(2)\right|^{2}\right\rangle} .
$$

Using Eqs. (98) and (101), one easily verifies property (2) cited in Sec. I:

$$
\int_{V_{a}} d^{6} z_{1} e_{1} \Gamma^{r}(1 \mid 2)+\int_{V_{a}} d^{6} z_{2} e_{2} \Gamma^{r}(2 \mid 1)=0
$$

Taking like-species interactions $\left(1=2\right.$, i. $\left.e_{,, s_{1}}=s_{2}\right)$, either of Eqs. (98) or (101) confirms property (1) of Sec. I, viz.,

$$
\left.\int_{V_{a}} d^{\sigma} z_{1} \Gamma^{r}(1 \mid 2)\right|_{1=2}=0
$$

However, since these results were derived in the Lorentz approximation, neither is strictly valid for like-particle interactions. To prove property (104) 
correctly, therefore, one must return to expression (89), and reevaluate it in the non-Lorentz case, where $M_{1} \sim M_{2}$. We shall see that the essential feature of Eqs. (98) or (101) needed for Eq. (104) to hold, namely the presence of the factor $\left(A_{1} / e_{1}-A_{2} / e_{2}\right)$, still holds for the case $M_{1} \sim M_{2}$.

Motivated by the form of Eqs. (103) and (104), therefore, we define the particle and energy fluxes, averaged over a toroidal shell $V_{a}$ :

$$
I_{n}(1 ; 2) \equiv \int_{V_{a}} d^{6} z_{1} \Gamma^{r}(1 \mid 2), I_{K}\left(1|2\rangle \equiv \int_{V_{a}} d^{6} z_{1} \frac{K_{01}}{T} \Gamma^{r}(1,2),\right.
$$

where $K_{01} \equiv K_{0}(1)$. C'sing Eqs. (64) and (89) in the definition of $I_{n}$, and again using the averaging effect of the $z$-integrations on the factors $J_{l}{ }^{2}$, one has

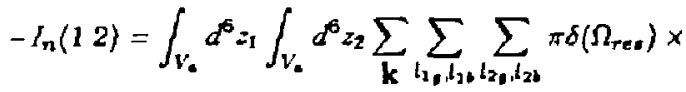

$$
\begin{aligned}
& \overline{J_{l q}^{2}} \overline{J_{l_{16}-\tau_{1} m^{2}}} \overline{J_{l_{2 q}}{ }^{2}} \overline{J_{l_{2 b}-\tau_{2} m^{2}}} \times \\
& \left\{\left.\left.4 \pi \bar{\alpha}\right|^{2} \epsilon^{r_{2}} \cdot l_{1}\left(l_{1} \cdot \epsilon^{r_{1}} \cdot A_{1}-l_{2} \cdot \epsilon^{r_{3}} A_{2}\right) f_{0}(1) f_{0}(2)\right|_{r_{1}}=r_{b 2}=r_{a} .\right.
\end{aligned}
$$

The new feature of the non-Lorentz case is that the term $\mathbf{l}_{\mathbf{2}} \cdot \boldsymbol{\Omega}_{2}^{\prime}$ in the argument $\Omega_{\text {res }}$ of the $\delta$-function cannot be neglected, thereby coupling the sums over $l_{1}$ and $l_{2}$. The full resonance condition is now

$$
0=\Omega_{\text {res }}=\omega_{g 1}-\omega_{g 2}+\omega_{b 1}-\omega_{b 2}+n\left(\Omega_{\zeta 1}-\Omega_{\zeta 2}\right),
$$

and the relevant ordering is

$$
\Delta \omega_{g 1} \sim \Delta \dot{u}_{g 2} \gg \Delta \omega_{b 1} \sim \Delta \omega_{b 2} .
$$

The new aspect of the problem is thus the evaluation of the I-sums in the presence of the full $\Omega_{\text {res }}$ :

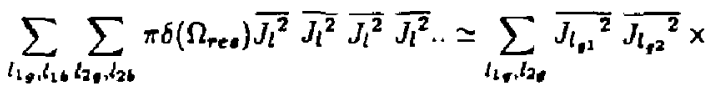

$$
\begin{aligned}
& \int d w_{b 2} \int d w_{b 1} \pi \delta\left(\Omega_{\text {rea }}\right) \frac{s\left(, \omega_{b 2}^{\prime}\right)}{\Delta \omega_{b 2}} \frac{s\left(, \omega_{b 1}^{\prime}\right)}{\Delta w_{b 1}} . \\
& =\pi \sum_{l_{1}, l_{2,}} \overline{J_{l_{31}}{ }^{2}} \overline{J_{l_{2}}{ }^{2}} I_{b}\left(\Delta w_{b 1}, \Delta w_{b 2}, \omega_{b 0}^{\prime}\right) \ldots,
\end{aligned}
$$

where $\omega_{b 0}^{\prime} \equiv \omega_{g 2}-\omega_{g 1}+m\left(\tau_{2} \Omega_{b 2}-\tau_{1} \Omega_{b l}\right)+n\left(\Omega_{\zeta 2}-\Omega_{\zeta 1}\right)$, and where the overlap integral $I_{b}$, given by

$$
I_{b}\left(\Delta \omega_{1}, \Delta \omega_{2}, x\right) \equiv \int_{-\infty}^{\infty} d \omega_{2} \frac{s\left(\Delta \omega_{2} / 2, \omega_{2}\right)}{\Delta \omega_{2}} \frac{s\left(\Delta \omega_{1} / 2, \omega_{2}-x\right)}{\Delta \omega_{1}}
$$


is even about $x=0$, and symmetric in $\Delta \omega_{1}, J_{2}$. With the definition $\Delta \omega_{>} \equiv \max \left(\Delta \omega_{1}, \Delta \omega_{2}\right)$, one has

$$
I_{b}()= \begin{cases}0 & x|>| \Delta \omega_{1}+\Delta \omega_{2} \mid / 2 \\ 1 / \Delta \omega_{>} & x|<| \Delta \omega_{1}-\Delta \omega_{2} \mid / 2\end{cases}
$$

and $I_{b}$ varies linearly with $x$ in the intervening interval $\mid \Delta \omega_{1}-\Delta \omega_{2} / 2<$ $x<: \Delta \omega_{1}+\Delta \omega_{2} \mid / 2$.

Owing to relations (108), the "short and broad" approximation used for the Lorentz case is still well satisfied, and thus, again, one has

$$
l_{b 1,2} \simeq \bar{l}_{b 1,2} \equiv \tau_{l, 2} m \text {. }
$$

As indicated by the spectrum in Eq. (67), the modes considered here have nearly zero frequency in the frame precessing at $\Omega_{\zeta E}$. Thus, for $M_{1} \sim M_{21}$ the dielectric function $\Delta_{a}\left(\omega=l_{2} \cdot \Omega_{2}\right)$ appearing in (106) again permits contributions oniy from terms with

$$
l_{g 1,2}=\bar{l}_{g}=0,
$$

causing the remaining sums over $l_{g 1}$ and $l_{g 2}$ in (109) to drop out. Using Eqs. $(109),(110),(112)$, and $(113)$ in $(106)$, therefore, one finds

$$
\begin{aligned}
& -I_{n}(1: 2)=\int_{V_{4}} d^{6} z_{1} \int_{V^{\prime}} d^{6} z_{2} \sum_{\mathbf{k}} \vec{Q}(1,2 \mid a) \times \\
& \epsilon^{r_{2}} \cdot \bar{I}_{1}\left(\bar{l}_{1} \cdot \epsilon^{r_{1}} \cdot A_{1}-\vec{l}_{2} \cdot \epsilon^{r_{2}} \cdot A_{2}\right) f_{0}(1) f_{0}(2) \\
& =\int_{V_{*}} d^{6} z_{1} \int_{V_{*}} d^{6} z_{2} \sum_{\mathbf{k}} \bar{Q}(1,2 ; a) n^{2}\left(\frac{-c}{B R b_{p}}\right)^{2} \times \\
& \frac{1}{e_{1}}\left(\frac{1}{e_{1}} A_{1}-\frac{1}{e_{2}} A_{2}\right) f_{0}(1) f_{0}(2)
\end{aligned}
$$

with kernel $\bar{Q}$ given by

$$
\left.\bar{Q}(1,2 \mid a) \equiv \pi \overline{J_{l, 2}{ }^{2}} \overline{J_{l_{g 2}}{ }^{2}} I_{b}\left(\Delta w_{b l}, \Delta w_{b 2}, w_{b 0}^{\prime}\right)|4 \pi \overline{\bar{\alpha}}|^{2}\right|_{I=\bar{l}},
$$

extending the Lorentz expression (93). The expression for $I_{K}(1 \mid 2)$ is the same as given in Eq. (114) for $I_{n}(1 \mid 2)$, but with an additional factor of $K_{01} / T$ in the integrand. The factor $\left(A_{1} / e_{1}-A_{2} / e_{2}\right)$ in expression (114), present also in the Lorentz results, implies Eq. (104), as already noted. In the Lorentz 
limit, the factor $I_{b}$ in (115) recovers the factor ${\overline{l_{b 1}-T_{1} m}}^{2} / \Omega_{b 1}=s\left(, \omega_{b 0}^{\prime}\right) / \Delta_{\omega_{b 1}}$ in Eq. (93). For the case $M_{1} \sim M_{2}, I_{b}\left(,, w_{60}^{\prime}\right)$ is a function of comparable size $\left(\sim \Delta \omega_{b 1}^{-1}\right)$ over a comparable range $\left(\sim \Delta \omega_{b 1}\right)$ of $\omega_{b 0}^{\prime}$ as in the Lorentz case, but with a somewhat modified functional form. The factor $\overline{J_{l, z}}$ in (115) is not present in (93), since there, all guroharmonics $l_{g 2}$ contributed, while only $l_{g 2}=0$ contributes in (115).

The k-summation in (114) may be performed using Eq. (96), yielding

$$
\begin{aligned}
-I_{n}(1 \mid 2)= & \int_{V_{*}} d^{6} z_{1} \int_{V_{c}^{\prime}} d^{6} z_{2} D^{\hat{\zeta}}(1 \mid 2) \frac{\left|u_{\tau}(2)\right|^{2}}{V_{a} \tau_{2}\left\langle\left|u_{\tau}(2)\right|^{2}\right\rangle}\left(\frac{-c}{B R b_{p}}\right)^{2} \times \\
& \frac{1}{e_{1}}\left(\frac{1}{e_{1}} A_{1}-\frac{1}{e_{2}} A_{2}\right) f_{0}(1) f_{0}(2)
\end{aligned}
$$

where the non-Lorentz kernel $\bar{Q}$ in Eq. (115) now yields a slightly modified diffusion coefficient $D^{<6}(1 \mid 2)$ from that in $(96)$ or $(75)$ :

$$
\begin{aligned}
D^{\zeta \zeta}(1 \mid 2) & =\pi \sum_{\mathbf{k}}|\overline{\bar{h}}(1 \mid \mathbf{k}, 2)|^{2} \overline{J_{l, 2}{ }^{2}} \overline{J_{l, z}{ }^{2}} I_{b} n^{2} \\
& =\left(\partial p_{\zeta} / \partial r_{b 1}\right)^{2} D_{R R}^{r r}(1 \mid 2)\left\langle\overline{J_{l_{1},}{ }^{2}} \overline{J_{l_{2},}{ }^{2}} I_{b} \Omega_{b 1}\right\rangle_{\mathbf{k}},
\end{aligned}
$$

with

$$
\left\langle\overline{J_{l_{1}}{ }^{2}} \frac{1}{J_{l_{2}}{ }^{2}} I_{b} \Omega_{b_{1}}\right)_{\mathbf{k}} \equiv \frac{1}{\pi \Delta k_{\perp} \rho_{g 1}} \frac{1}{\pi \Delta k_{\perp} \rho_{g 2}} \frac{1}{\pi \Delta k_{\perp} r_{1}}\left[\begin{array}{c}
\pi^{2} / 32 \\
1 / 2
\end{array}\right] \sqrt{\frac{\pi}{2}},
$$

and where as in (76), the upper(lower) component holds for $\tau=0(1)$.

We now turn to the Onsager symmetries of the transport coefficients. In the following, it is convenient to use indices $p$ or $q$, which may take on values $n$ and $K$ in designating the components of the fluxes $I_{p}$ and forces $A_{p}$. We define the coefficients of the thermal force terms, which can be read off from Eq. (114), and from its counterpart for $I_{K}(1 \mid 2)$ :

$$
\begin{aligned}
L_{p q}^{12} \equiv & \frac{1}{e_{1}^{2}}\left(\frac{-c}{B R b_{p}}\right)^{2} \int_{V_{*}} d^{6} z \int_{V_{*}} d^{6} z^{\prime} f_{0 z_{1}}(z)\left[\frac{K_{0}(z)}{T}\right]^{x_{p}+x_{q}} \times \\
& f_{0 \mu_{2}}\left(z^{f}\right) \sum_{\mathbf{k}} \bar{Q}\left(z, z^{\prime} \mid a\right) n^{2} \\
M_{p q}^{12} \equiv & \frac{1}{e_{1} e_{2}}\left(\frac{-c}{B R b_{p}}\right)^{2} \int_{V_{*}} d^{6} z \int_{V_{*}} d^{6} z^{\prime} f_{0 \mu_{1}}(z)\left[\frac{K_{0}(z)}{T}\right]^{x_{p}} \times \\
& f_{0 u_{2}}\left(z^{\prime}\right)\left[\frac{K_{0}\left(z^{\prime}\right)}{T}\right]^{x_{q}} \sum_{\mathbf{k}} \bar{Q}\left(z, z^{\prime}(a) \pi^{2} .\right.
\end{aligned}
$$


The $L_{p q}^{12}$ are the coefficients arising from $\mathbf{D}$, and the $M_{p q}^{12}$ are those arising from $F$. We have used $z$ and $z^{\prime}$ for the variables of integration here instead of $z_{1}$ and $z_{2}$ used previously to emphasize that the species labels 1 and 2 in the superscripts of $L_{p q}^{12}$ and $M_{p q}^{12}$ correspond to the species labels $s_{1}, s_{2}$ on the distribution functions, and not to the integration variables. This aroids ambiguity when $s_{1}=s_{2}$. The exponent $x_{p}$ of the energy-weighting equals $0(1)$ for $p=n,(K)$, and similarly for $x_{q}$. In terms of the $L$ 's and $M$ 's, one can write $I_{n}(1 \mid 2)$ and $I_{K}(1 ' 2)$ succinctly as

$$
-I_{p}(1 \mid 2)=\sum_{q=n, K}\left(L_{p q}^{12} \cdot A_{q 1}-M_{p q}^{12} A_{q 2}\right),(p=n, K) .
$$

From inspection of Eqs. (119) and (120), one notes the following symmetries:

$$
L_{p q}^{12}=L_{q p}^{12}, M_{p q}^{12}=M_{q p}^{21}, e_{1} L_{p n}^{12}=e_{2} M_{p n}^{12}
$$

but

$$
e_{1} L_{p K}^{12} \neq e_{2} M_{p K}^{12}
$$

These relations may be used to efficiently prove Eqs. (103) and (104), as well as the Onsager symmetries, now under consideration.

Following essentially the same steps as used to obtain $I_{n}$ in Eq.(114), one may evaluate expression (16) for $\dot{S}$. The result is

$$
\begin{aligned}
\dot{S}= & \frac{1}{2} \sum_{r_{\mathbf{a}}} \sum_{1,2} \int_{v_{\mathbf{a}}} d^{6} z_{1} \int_{V_{a}} d^{6} z_{2} f_{0}(1) f_{0}(2)\left(\frac{-c}{B R b_{p}}\right)^{2}\left(\frac{1}{e_{1}} A_{1}-\frac{1}{e_{2}} A_{2}\right)^{2} \times \\
& \sum_{\mathbf{K}} \overline{\bar{Q}}(1,2 \mid a) n^{2} \\
= & -\frac{1}{2} \sum_{r_{0}} \sum_{1,2}\left[A_{n 1} I_{n}(12)+A_{n 2} I_{n}(2 \mid 1)+A_{K 1} I_{K}(1 \mid 2)+A_{K 2} I_{K}(1 \mid 2)\right] \\
= & -\sum_{r_{0}} \sum_{1,2} \sum_{p} A_{p 1} I_{p}(12) .
\end{aligned}
$$

The first form given parallels expression (114). The four terms in the second form come from identifying the coefficients of each of the four terns in the first thermal-force factor $A_{1} / e_{1}-A_{2} / e_{2}$ on the first line, and the final version presents the second form somewhat more compactly. The total particle and energy fluxes for species 1 are given by $I_{p}(1) \equiv \sum_{2} I_{p}(1 \mid 2)$. Assuming the thermal forces $A_{q}$ are independent of species, the total particle and energy 
fluxes (summed over species 1) are given by

$$
\cdots I_{p} \equiv-\sum_{1,2} I_{p}(1 \mid 2)=\sum_{q} L_{p q} A_{q},
$$

where from Eq.(121), $L_{p q} \equiv \sum_{1,2}\left(L_{p q}^{12}-M_{p q}^{12}\right)$. Using (124) in (123), $\dot{S}$ may be written in the symmetric form ${ }^{16}$

$$
\dot{S}=\sum_{r_{a}} \sum_{p \cdot q} A_{p} L_{p q} A_{q} .
$$

The Onsager symmetries are expressed in the relations

$$
L_{\mathbf{p q}}=L_{\mathbf{q p}}
$$

Since we are considering only a $2 \times 2$ matrix $L_{p q}$ here, the only nontrivial member of relations (126) is $L_{n K}=L_{K n}$. This is easily demonstrated, using the definition of $L_{p q}$, and the first two of the symmetries (122).

\section{Discussion}

In previous sections, we have evaluated both the diffusive and frictional portions of the radial fluxes, for a particular turbulent transport problem of interest. The evaluation has been carried out for each of the three cases when the mass of the scattered species is much less than [Eq. (98)], much greater than [Eq. (101), and equal to [Eq. (116)] that of the scattering species. For each of these, both portions of the flux are simply given in terms of the corresponding diffusion coefficient $D^{r}(1 \mid 2)$. Here, we assess the physical implications of these expressions, by considering the relative sizes and scalings of $D^{r+}(1 / 2)$ for all four possible species-species interactions.

We use $\langle 1\rangle_{\mathbf{k}}$ as shorthand notation for the orbit-averaging factor

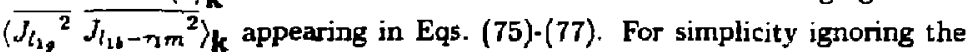
factor $\overline{J_{21}{ }^{2}}$ entering the comparable-mass expression $D^{<\zeta}(1 \mid 2)$ in Eq. (117), as it is of secondary importance, the remaining angle-bracketed term is approximately equal to $\langle 1\rangle_{k}$ as well. Then the expressions for $D^{r}(1: 2)$ for all cases may be approximately written

$$
D^{r}(1 \mid 2) \simeq \frac{\pi}{\Omega_{b>1}}\langle\rangle_{k} u^{u^{2}}(1) \dot{b}^{2}(2) .
$$

Consistent with the definition of $\Delta w_{>}$in Eq. (111), the symbol " $>$" here refers to the species with larger thermal speed $v_{T}$ (and so smaller mass). 
Expression (127) transparently reduces to Eq. (77) for the case $M_{1} \ll M_{2}$ to which $(98)$ pertains, and also approximately yields the equal-mass case (117), as already noted. For the case $M_{2} \gg M_{1}$ described by Eq. (101), approximately evaluating expression (102), one finds $D^{r r}(2 \mid 1)$ given by Eq. (127), with $\bar{b}^{2}(1)$, representing the perturbing fields driven by species 1 , given by

$$
\bar{b}(1) / \bar{b}(2)]^{2} \simeq\left(n_{1} e_{1}^{2} v_{T 1}^{2}\right) /\left(n_{2} e_{2}^{2} v_{T 2}^{2}\right) \text {. }
$$

Expression (128) arises from our adoption of Eqs. (90) and (91) to describe the coupling $\overline{\bar{\alpha}}(1,2)$ between any two species 1 and 2 . Applying expression (127), one finds

$$
\begin{aligned}
D^{r r}(1 \mid 1): & D^{r r}(1 \mid 2): D^{r r}(2 \mid 1): D^{r r}(2 \mid 2) \\
& :: u^{2}(1) \dot{b}^{2}(1) \frac{\langle 1\rangle_{\mathbf{k}}}{\Omega_{b 1}}: u^{2}(1) \bar{b}^{2}(2) \frac{\langle 1\rangle_{\mathbf{k}}}{\Omega_{b 1}}: u^{2}(2) \dot{b}^{2}(1) \frac{\langle 1\rangle_{\mathbf{k}}}{\Omega_{b 1}}: u^{2}(2) \dot{b}^{2}(2) \frac{\langle 2\rangle_{\mathbf{k}}}{\Omega_{b 2}} \\
& : n_{1} e_{1}^{2} v_{T 1}^{4}: n_{2} e_{2}^{2} v_{T 1}^{2} v_{T 2}^{2}: n_{1} e_{1}^{2} u_{T 1}^{2} v_{T_{2}}: n_{2} e_{2}^{2} v_{T 2}^{4} \frac{\langle 2\rangle_{\mathbf{k}} \Omega_{b 1}}{\langle 1\rangle_{\mathbf{k}} \Omega_{b 2}}
\end{aligned}
$$

and thus, for $M_{1} \ll M_{2}$,

$$
n_{1} e_{1}^{2} D^{r r}(1,1) \gg n_{1} e_{1}^{2} D^{r r}(1,2) \simeq n_{2} e_{2}^{2} D^{r r}(2 \mid 1) \gg n_{2} e_{2}^{2} D^{r r}(2 ; 2)
$$

The statement $n_{1} e_{1}^{2} D^{r r}(1 \cdot 2) \simeq n_{2} e_{2}^{2} D^{r r}(2 \mid 1)$ here is the approximate counterpart of statement (103) of intrinsic ambipolarity. Because $D^{\text {rr }}(1 \mid 1) \gg$ $D^{\text {rr }}(1 ; 2)$, the energy flux of species 1 , which is dominated by $\left.D^{\text {rr }}(1) 1\right)$, will be much more rapid than the particle flux, to which only $D^{r r}(12)$, and not $D^{r s}(1 \mid 1)$, contributes. Both the particle and energy fluxes of species 2 due to this mechanism will be dominantly governed by $D^{\text {rr }}(2 \mid 1)$. One notes that the situation is quite aralogous to the relations holding for collisional (neoclassical) transport, except that for that mechanism, the roles of the heavier and lighter species are interchanged. This is because collisional transport is an electrostatic mechanism, and thus lacks the velocity-weighting in both the factors $u^{2}$ and $\bar{b}^{2}$ in Eq. (127), which in the present the magnetic mechanism enhances the transport and fluctuation spectrum of the higher-velocity species.

This completes the demonstration of the unity which exists between the different tokamak transport mechanisms, facilitated by use of the gBL operator. Extension of the properties demonstrated above for the case of magnetic turbulence (and already well-established for syrumetric transport) to other cases of interest (for example, electrostatic turbulence, or internally generated ripple) should be straightforward. For the sample turbulent mechanism 
chosen, we have obtained an explicit expression for the anomalous pinch term, and showed that, because of its close relation to the contribution frori the diffusive term, the tota' fux possesses the appropriate conservation laws, which are lost by test-particle calculations. Moreover, we have seen that, just as for symmetric transport, neglect of this term can totally modify the expected particle flux for one species from the correct answer.

The theory developed here is incomplete. Possible modifications in the transport results may result rion using more realistic descriptions of the mode structure, including the appropriate combination of electrostatic versus electromagnetic components. The treatment of the non-Lorentz case given here in Sec. $V$ is only for the particular turbulent mechanism studied, and applied principally toward treatment of the equal-mass case. The effect of the DC inductive electric field has been ignored here, resulting in only a $2 \times 2$ Onsager matrix, rather than the $3 \times 3$ matrix of a full theory. Some work in incorporating this extension into the action-angle transport framework has already been carried out in Ref. 6, for a test-particle calculation, and an extension of this to the present theory should be possible. Finally, we again note that the application of the thermal structure of the gBL operator to fully turbulent transport is somewhat ad hoc. Thus, for example, it is unclear that our demonstration of the Onsager relations for this theory, which depended on the use of the thermal form (90) of the particleparticle coupling, can be extended to a fully turbulent theory. However, our "pseudo-thermal" ansatz does yield an analytically manageable theory with the requisite symmetries, conservation laws, and features of self-consistency, and which becomes fully valid in the limit of a stable plasma, where the dielectric function $\Delta_{a}$ reverts to its usual thermal form. Thus, the theory represents an improvement upon the traditional quasilinear approach, adding to that approach several of the important properties required of a complete theory.

\section{Acknowledgment}

This work supported ty U.S. Department of Energy Contract No.DEAC02-76-CHO3073. 


\section{References}

${ }^{1}$ H. E. Mynick, Princeton Plasma Physics Laboratory Report PPPL-2458 (1987)(accepted by J. Plasma Physics).

${ }^{2}$ A. N. Kaufman, Phys. Fluids 15, 1063 (1972).

${ }^{3}$ P. H. R.utherford, Phys. Fluids 13, 482 (1970).

${ }^{4}$ P. C. Liewer, Nucl. Fusion 25, 543 (1985).

${ }^{5}$ H. E. Mynick and J. A. Krommes, Phys. Fluids 23, 1229 (1980),

${ }^{6}$ R. D. Hazeltine, S. M. Mahajan and D. A. Hitchcock, Phys. Fluids 24, 1164 (1981).

${ }^{7}$ H. E. Mynick, Nucl. Fusion 26, 491 (1986).

${ }^{8}$ A. N. Kaufman and T. Nakayama, Phys. Fluids 13, 956 (1970).

${ }^{9}$ R. E. Duvall and H. E. Mynick, Proc. 1988 Sherwood Theory Conference, $2 \mathrm{C} 28$ (Gatlinburg, TN)(to be published).

${ }^{10}$ F. L. Hinton and R. D. Hazeltine, Rev. Mod. Phys 48, Part 1, 239 (1976).

${ }^{11}$ K. C. Shaing, Oak Ridge National Laboratory Report ORNL/P-87/3477 (1987)(submitted for publication).

${ }^{12}$ A. B. Rechester and M. N. Rosenbluth, Phys. Rev. Letters 40, 38 (1978).

${ }^{13}$ S. V. Putvinskii and R. V. Shurygin, Fiz. Plasmy 10, 933 (1984).

${ }^{14}$ V. Ya. Goloborod'ko, Ya. I. Kolesnichenko and V. A. Yavorskii, Physica Scripta T16, 46 (1987).

${ }^{15}$ R. J. Goldston, R. B. White and A. H. Boozer, Phys. Rev, Letters 47, 647 (1981).

${ }^{16}$ M. N. Rosenbluth,R. D. Hazeltine and F. L. Hinton, Phys. Fluids 15, 116 (1972). 


\section{Figures}

FIG. 1. Illustration of the summation/integration to be performed over the $\left(l_{g}, l_{b}\right)$ or $\left(\omega_{g}, \omega_{b}\right)$-plane, in the evaluation of the gBL operator. Indicated is the resonance line $\Omega_{\text {res }}=0$, along which the integration contributes, and the rectangular box, of width $\Delta w_{g}$ and height $J w_{b}$, within which the integrand is appreciable. The dots within this rectangle indicate the position of resonance with indjvidual Fourier components $l_{1}$ of the perturbing Hamiltonian of the scattering test-particle. 
* B8T0047

i

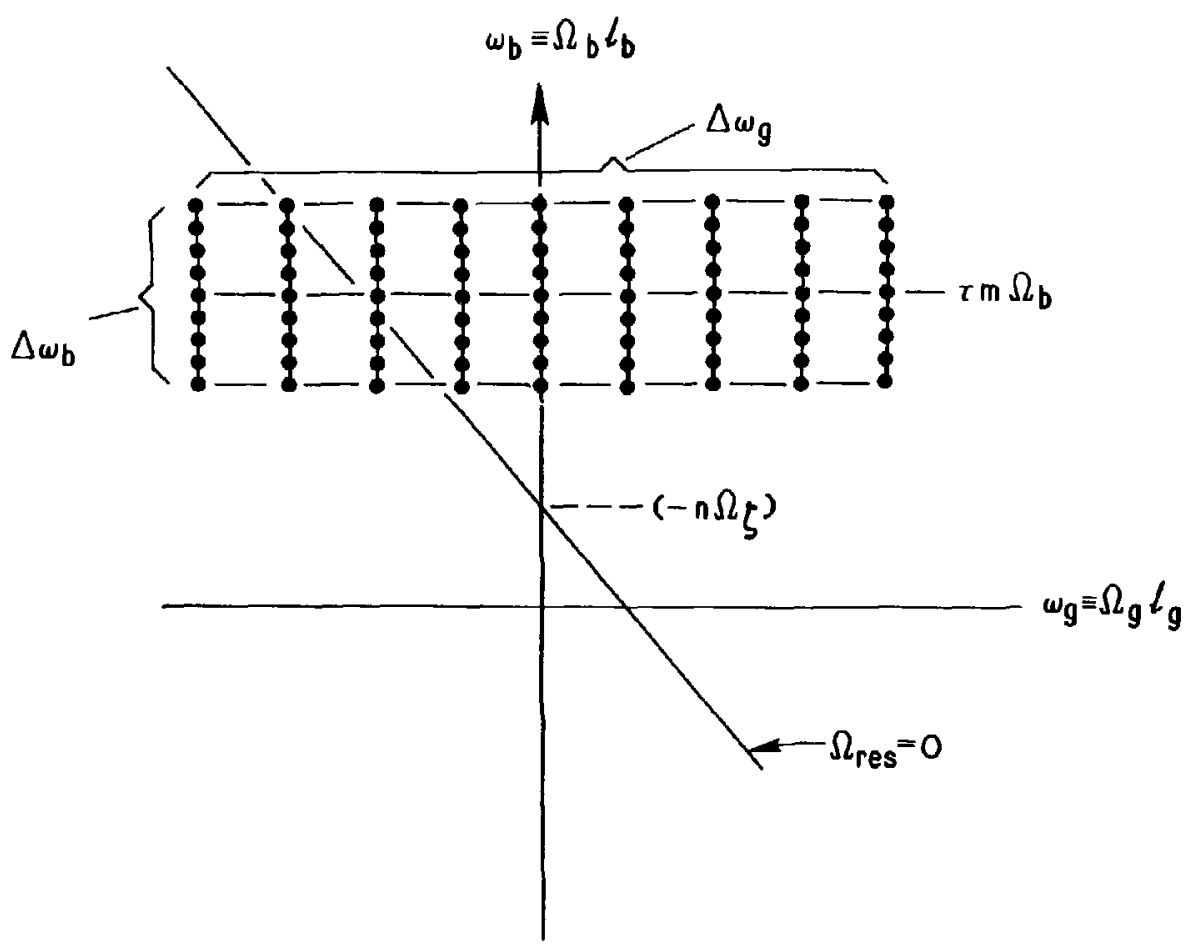


Dr. Fronk J. Paoloni, Univ of Wallongong. AuSTRALIA Prof, M.H. Brennan, Univ Sydney, AuSTRALIA

Plasma Research Lab., Australian Nat. Univ., AUSTRALIA

Prof. I.R. Jones, FI inders Univ, AUSTRALIA

Prof. F. Cap, Inst Theo Phys, Austria

Prof. M. Heindler, Institut fur Theoretische Physik, AUSTRIA

H. Goossens, Astronomisch Instituut, BELGIUM

Ecole Royale Militaire, Lab de Phys Plasmas, BELGIUM

Commission-European, Dg-XII Fusion Prog, BELGILM

Prof. R. Bouciaue, Laboratorium voor Natuurkunde, BELGIUM

Dr. P.H. Sakanaka, Instituto Fisica, BRAzIL

Instituto de Pesquisas Espociasi-INFE, BRAzIL

Documents office, Atomic Energy of Canada Limited, CANaDa

Dr. M,P. Bachynski, MPG Technologies, InC., CANADA

Or. H.M. Skarsgard, University of Soskatctiewan, CANAOA

Dr. H. Barnard, University of British Columbia, CANADa

Prot. J. Teichmann, Univ. of Montred, CANADA

Prot. S.R. Sreenivasan, University of Calgary, CANADA

Prot. Tudor W. Johnston, INRS-Energie, CANADA

Dr. C.R. Janes, Univ, of Alberta, CANADA

Dr. Poter Lukac, Komenskeho Uniy, CZECHOSLOYAKJA

The Librarian. Cuinam Laboratory. ENGLAND

The Librarian, Rutherford Apoleton Laboratory, ENGLAND

Mrs. S.A. Huteninson, JET Library, ENGLAND

C. Mouttet, Lab. de Physique des Milieux ton isés, FRance

J. Radet, CEN/CADARACHE - gat 506, FRANCE

Univ. of Ioannina, Library of Physics Dept. GREECE

Or. Tom Mual, Açadeny Gibliographic Ser., HONG KONG

Preprint Library. Hungar ian Acacemy of Sciences. HUNGARY

Dr. B. Dasqudta, Saha Inst of Nucl. Phys.. INDIA

Dr. P. Xaw, Institute for PIasma Reseorch, IND|A

Dr. Philip Rosenau, Isrdel Inst. Teen, ISRAEL

Librarian, Int'I Ctr Theo Phys, ITALY

Prof. G. Rostagni, Jni Di Padova, ITALY

Miss Clelia De Palo, AsSOC EURATOH-ENEA, ITALY

Biblioteca, Instituta di fisica del PIasma, ITALY

Or. H. Yamato. Toshida Res \& Dev, JAPAN

Prot. I. Kawakani, Aromic Energy Res. Institute. JAPAN

Prof. Kyoji Nishikana, Univ of Hiroshime, JAPAN

Direc. Dept. Large Tokamak Res. JAERI, JAPAN

Prof. Satoshi Itoh, Kyushu University, JAPAN

Research Info Center. Nagora University, JAPAN

Prof. S. Tanaka, Kyoto University, JAPAN

Library, Kyoto university, JAPAN

Prof. Nobuyuki Inoue, University of Tokyo, JAPAN

5. Mor $i$, JAERI, JAPAN

Librarian. Korea Advanced Energy Res. Institute, KOREA

Prot. 0.1. Choi, Adv. Inst Sci s Tech, KOREA

Prot. 0.S. Liloy, University of Waikoto, NEW ZEALAND

Institute of Plasma Physics, PEOPLE'S REPU⿴囗IC OF CHINA

Librarian, Instifute of Phys., PEOPLE'S REPUBLIC OF CHINA

Library. Tsing Hua University, PEOPLE'S REPUBLIC OF CHINA
2. Li, Southwest Inst. Physics, PEOPLE'S REPU⿴LIC OF CHIy' Prof. J.A.C. Cabral, Inst Superior Tecnico, RORTUGAL

Dr. Octavian Petrus. AL I CUZA University, ROMANIA

Dr. Johan de Villiers, Fusion Studies, AEC, SO AFRICA

Prof. M.A. Hellberg, University of Natal, SO AFRIC:

C.I.E.M.A.T., Fusion Div. Library, SPAIN

Dr. Lennart Stenflo, University of UMEA, SWEDEN

Library, Royal Inst Tech, SWEDEN

Prof. Hans Wilnelmson, Chalmers Uniy Tech, SWEOEN

Centre Phys des PIasmas, Ecole Polytech Fed, SwitzERLAND

Bibl iotheek, Fom-Inst yoor Plasma-fysica, THE NETHERLANDS

Or. D.D. Ryutov, Siberian Acad Sei, USSR

Or. G.A. Eliseev, Kurchatov Instituta, USSR

Dr. V.A. Glukhikh. Inst Electrophysical Apparatus, USSR

Dr, V.T. Tolok, inst. Phys. Tech, USsR

Dr. L.M. Kovrizhnykh, Institute Gen. Physics, USSR

Nuclear Res. Estadi ishment, Jul ich btd., W. GERManY

Bialiothek, Inst. Fur PIasmatorschung, W. GERMANY

Dr. K. Schindler, Runr Univers itat Bochum, $*$. GERMANY

ASOEX Resding Rm, IPP/Max-Planck-Institut fur

Plasmannysik, W. GERMANY

Librarian, Max-Planck Institut, W. GERMANY

Prot. R.K. Janey, inst Phys, Yugoslavia 